Pacific Journal of Mathematics

ON THE INVARIANT MEAN ON TOPOLOGICAL SEMIGROUPS 


\title{
ON THE INVARIANT MEAN ON TOPOLOGICAL SEMIGROUPS AND ON TOPOLOGICAL GROUPS
}

\section{EDMOND GRANIRER}

\begin{abstract}
Let $S$ be a topological semigroup and $C(S)$ be the space of bounded continous functions on $S$. The space of translation invariant, bounded, linear functionals on $C(S)$ and its connection with the structure of $S$, are investigated in this paper. For topological groups $G$, not necessarily locally compact, the space of bounded, linear, translation invariant functionals, on the space $U C(G)$ of bounded uniformly continuous functions, is also investigated and its connection with the structure of $G$ pointed out. The obtained results are applied to the study of the radical of the convolution algebra $U C(G)^{*}$ (for locally compact groups, or for subgroups of locally convex linear topological spaces) and some results which seem to be unknown even when $G$ is taken to be the real line are obtained.
\end{abstract}

The topological semigroup $S$ is assumed to have a separately continuous multiplication, and $C(S)$ is given the usual sup norm. $C(S)^{*}$ will denote the conjugate Banach space of $C(S)$. If $a \in S$ and $f$ is any function on $S$ then $f_{a}$ is defined by $f_{a}(s)=f(a s)$ for $s \in S . \varphi \in C(S)^{*}$ is said to be left invariant if $\varphi\left(f_{a}\right)=\varphi(f)$ for each $f$ in $C(S)$ and $a$ in $S . \quad J_{c} l(S)$ will denote the space of left invariant elements of $C(S)^{*}$. A topological semigroup is said to be left amenable as a discrete semigroup if there is a linear functional $\varphi \neq 0$ on $m(S)$ (the space of all real bounded functions on $S$ with the usual sup. norm) which satisfies $\varphi\left(f_{a}\right)=\varphi(f)$ for each $a$ in $S$ and $f$ in $m(S)$ and $\varphi(f) \geqq 0$ if $f \geqq 0$. An analogous definition holds for the right amenable case. A topological semigroup is said to be amenable as a discrete semigroup if it is right and left amenable as a discrete semigroup.

The following are results of I. S. Luthar [12]:

(1) If $S$ is an abelian topological semigroup with a compact ideal then $\operatorname{dim} J_{c} l(S)=1$

(2) If $G$ is an abelian topological group having a certain property $P$ (Any noncompact locally compact group or any nonzero subgroup of a linear convex topological vector space has this property see [12] p. 406) then $\operatorname{dim} J_{c} l(G) \geqq 2$.

We say that a subset $S_{0}$ of the semigroup $S$ is a left-ideal group if $S_{0}$ is a group when endowed with the multiplication induced from $S$

Received December 2, 1963. Supported in part by National Science Foundation Grant 19869. 
and $s s_{0}$ belongs to $S_{0}$ for any $s$ in $S$ and $s_{0}$ in $S_{0}$. If $S$ is also a topological space then $S_{0} \subset S$ is a compact left-ideal group if it is a left-ideal group and a compact subset of $S$.

The following theorem is proved in Ch. IV of this paper:

THEOREM IV-1. Let $S$ be a topological semigroup (with only separately continuous multiplication and no separation axioms) containing exactly $n(0<n<\infty)$ compact left-ideal groups. Then $\operatorname{dim} J_{c} l(S)=n$.

If $S$ is abelian and contains a compact ideal then as known and directly shown, $S$ contains a unique group and compact ideal (see the argument in [12] at the top of p. 404) and so $\operatorname{dim} J_{c} l(S)=1$, which yields Luthar's first result.

When considering this Theorem IV-I one is tempted to conjecture that its converse if true i.e.

(A) If $S$ is a topological semigroup and $\operatorname{dim} J_{c} l(S)=n 0<n<\infty$, then $S$ contains exactly $n$ compact left-ideal groups ${ }^{1}$.

This conjecture, even when allowing $S$ to be a topological semigroup with jointly continuous multiplication and $S$ to be a Hausdorff regular topological space, cannot be true as the following simple example shows:

E. Hewitt (see [22]) has constructed a regular Hausdorff space $S$ such that the only real continuous functions on it are the constant functions. Define in this space $S$ the following multiplication: $a b=a$ for any $a, b \in S$. If $F: S \times S \rightarrow S$ is defined by $F(a, b)=a b=a$ and $U \subset S$ is open then $F^{-1}(U)=\{(a, b) ; a b \in U\}=\{(a, b) ; a \in U\}=U \times S$ which is surely open in $S \times S$. Therefore multiplication in $S$ is jointly continuous and $S$ is a Hausdorff regular topological space. But $C(S)$ is one dimensional and so $C(S)^{*}$ is one dimensional. Moreover, if we define $\varphi(f)=f(a)$ for each $f$ in $C(S)$ and some fixed $a \in S$ then $\varphi \neq 0$ is easily seen to be left invariant. Thus $\operatorname{dim} J_{c} l(S)=1$. But $S$ does

1 This conjecture made by I. S. Luthar for the abelian case (see [12] p. 403) and believed to be true by this author for completely regular topological semigroups, is not true even for abelian topological groups. In fact let $G$ be a pseudocompact noncompact abelian topological group and $A$ a translation invariant nonnegative linear functional on $C(G)$ such that $\|A\|=1$. By Theorem 4.1 of W. Comfort and K. Ross (see [23] $G$ ) is totally bounded and each $f$ in $C(G)$ is uniformly continuous and therefore has a unique uniformly continuous extension $\bar{f}$ to the compact topological group $\bar{G}$ (the completion of $G)$. Conversely any $\bar{f} \in C(\bar{G})$ is the uniformly continuous extension of a unique $f \in C(G)$. Define now the linear functional $\bar{A}$ on $C(\bar{G})$ by $\bar{A} \bar{f}=A f$. It is not hard to show now, after using heavily the Comfort-Ross theorem, that $\bar{A}$ is translation invariant (with respect to the elements of $\bar{G}$ ) nonnegative and $\|\bar{A}\|=1$. Therefore $\bar{A} \bar{f}=\int \bar{f} d m$ where $m$ is the unique normalized Haar measure on $\bar{G}$. This shows that $\operatorname{dim} J_{c} l(G)=1$ while $G$ is not compact. Many thanks are due to W. Comfort and K. Ross for kindly letting this author have a preprint of their paper. 
not contain any proper left ideal since $S b=S$ for any $b \in S$. And $S$ is neither a group nor is it compact (For a compact hausdorff space $S, C(S)$ even separates points). Nevertheless, in certain cases, statement (A) holds true. The following theorem is proved in Ch. II of this paper:

THEOREM II-2. Let $S$ be a countable topological semigroup which is left amenable as a discrete semigroup and which is a $T_{1}$ regular topological space (and therefore completely regular).

Then $\operatorname{dim} J_{c} l(S)=n, n<\infty$, if and only if then $S$ contains exactly $n$ finite left-ideal groups 2 .

Consider now $G$ to be a topological group and denote by $L U C(G) \subset C(G)$ the space of left uniformly continuous functions on the group $G$. Let $J_{u} l(G) \subset L U C(G)^{*}$ be defined as:

$$
\left\{\varphi ; \varphi\left(f_{a}\right)=\varphi(f) \text { for each } f \text { in } L U C(G) \text { and } a \text { in } G\right\} \text {. }
$$

Also, recall that at least any abelian or solvable or locally finite group $G$, is left amenable as a discrete group. (see Day [4] for these and more examples). We can now state our next result:

THEOREM III-2. Let $G$ be a separable locally compact hausdorff topological group which is amenable as a discrete group. Then

(1) Either $\operatorname{dim} J_{u} l(G)=1$ or $\operatorname{dim} J_{u} l(G)=\infty$ and $\operatorname{dim} J_{u} l(G)=1$ if and only if $G$ is compact.

(2) Either $\operatorname{dim} J_{c} l(G)=1$ or $\operatorname{dim} J_{c} l(G)=\infty$ and $\operatorname{dim} J_{c} l(G)=1$ if and only if $G$ is compact.

THEOREM III-3. Let $G$ be any separable (not necessarily closed) subgroup of locally convex linear topological space. Then

(1) Either $\operatorname{dim} J_{u} l(G)=1$ or $\operatorname{dim} J_{u} l(G)=\infty$ and $\operatorname{dim} J_{u} l(G)=1$ if and only if $G=\{0\}$.

(2) Either $\operatorname{dim} J_{c} l(G)=1$ or $\operatorname{dim} J_{c} l(G)=\infty$ and $\operatorname{dim} J_{u} l(G)=1$ if and only if $G=\{0\}$.

From these theorems it is obvious that for both the considered groups $\operatorname{dim} J_{u} l(G)=\operatorname{dim} J_{c} l(G)$ invariably holds. An example of a countable abelian topological group in which $\operatorname{dim} J_{u} l(G)=1$ while $\operatorname{dim} J_{c} l(G)=\infty$ is given in Ch. III. This example uses heavily the theorems on countable topological semigroups obtained in Ch. II.

Separable topological groups $G$ which are amenable as discrete groups and have a certain property $B(G$ has property $B$ means that $G$ admits

2 One cannot hope for much more than this theorem. In fact an example of a locally compact abelian topological semigroup (with jointly continuous multiplication) for which statement $A$ does not hold true for any $n$ can be given. 
a real left uniformly continuous unbounded function. Noncompact locally compact groups, nonzero subgroups of locally convex linear topological spaces and groups which admit a right invariant unbounded metric have this property.) are considered in Ch. III and for them it is proved that $\operatorname{dim} J_{u} l(G)=\infty$ and $\operatorname{dim} J_{c} l(G)=\infty$ (see Theorem (III. 1)). It should be remarked here, that our results neither imply, nor are implied by Luthar's results in [12]. They improve Luthar's results in the case where $G$ is separable and either locally compact or a subgroup of a locally convex linear topological space (and also in certain other cases) but they do not deal at all with the non separable case.

We consider further in this paper the Banach space $L U C(G)^{*}$ (i.e. the conjugate of $L U C(G)$ ). As known and easily seen $L U C(G)^{*}$ becomes a Banach algebra under convolution as multiplication (while convolution in $C(G)^{*}$ cannot generally be defined, as known). If we denote by $R(G)$ the radical of the Banach algebra $L U C(G)^{*}$ (which may not be commutative though $G$ is so) then the following results are obtained, as immediate consequences of our work:

THEOREM. If $G$ is a separable, noncompact, locally compact topological group which is amenable as a discrete group, then the radical $R(G)$, of $L U C(G)^{*}$ is infinite dimensional (see Theorem III-6)

Combining this theorem with a known result, to be found in Rudin [15], which asserts that if $G$ is compact abelian then $C(G)^{*}$ is semisimple one gets.

THEOREM III-4. Let $G$ be a separable abelian locally compact topological group. Then either $R(G)=\{0\}$ or $R(G)$ is infinite dimensional. Moreover $R(G)=\{0\}$ if and only if $G$ is compact ${ }^{3}$.

THEOREM III-5. Let $G$ be a separable subgroup of a locally convex linear topological space. Then either $R(G)=\{0\}$ or $R(G)$ is infinite dimensional. Moreover $R(G)=\{0\}$ if and only if $G=\{0\}$.

If we take $G$ to be the real line $R$ and therefore $L U C(G)=U C(R)$ to be the space of real uniformly continuous bounded functions on $R$ then the algebra $U C(R)^{*}$, with convolution as multiplication, has as infinite dimensional radical. It is not hard to see that this holds true also for the complex valued uniformly continuous functions on $R$. Even this result for the real line seems to be unknown.

${ }^{3}$ It can be proved that $R(G)=\{0\}$ for any compact topological group $G$. Therefore Theorem III-4 holds true for any separable locally compact $G$, which is amenable as a discrete group. Thanks are due to Professor M. Rajagopalan for communicating this fact to me. 
In the end it is a pleasure for me to thank Ranga $R$. Rao for the friendly and fruitful conversations I had with him. It was in fact his idea to use the functions $\left\{f_{n}\right\}$ in the proof of Theorem III-1.

Some notations. $S$ is a topological semigroup if it has an associative multiplication and is a topological space (with no separation axioms) and for any fixed $a$ in $S$ the mappings $s \rightarrow a s$ and $s \rightarrow s a$ are continuous from $S$ to $S$. (i.e. multiplication is only separately continuous). We do not assume that $(x, y) \rightarrow x y$ from $S \times S \rightarrow S$ is continuous. As remarked in [19] p. 64 the multiplicative semigroup or linear continuous operators on a Banach space with the weak operator topology is only separately continuous.

$G$ is a topological group if it is a group, has a Hausdorff topology and $(x, y) \rightarrow x y^{-1}$ from $G \times G \rightarrow G$ is continuous (i.e. in this case jointly continuous multiplication.)

If $S$ is a set then $l_{1}(S), m(S)$ are defined as usual (see Day [5] p. 28) and if $S$ has a topology then $C(S)$ is again defined as usual (see introduction). We stress that we deal only with real valued bounded functions in this paper. If $X, Y$ are normed spaces then $X^{*}, Y^{*}$ are their respective conjugate Banach spaces and if $T: X \rightarrow Y$ is linear then $T^{*}: Y^{*} \rightarrow X^{*}$ denotes the conjugate of $T$ (see [5] pp. 14-17.)

If $A \subset S$ then $1_{A}$ is the function whose value is one an $A$ and zero otherwise (when no ambiguity may arise, 1 will denote the constant one function on $S$, i.e. $1_{s}$ ). If $A, B$ are subsets of $S$ then $A-B$ will invariably mean the set of points of $A$ which are not in $B$.

If $f$ is a function on $S$ and $a \in S$ then $f_{a}, f^{a}$ are defined by $\left(f_{a}\right)(s)=f(a s)$ and $\left(f^{a}\right)(a)=f(s a)$ for each $s$ in $S$. A linear manifold (which means the same as a linear subspace or in short a subspace) $L \subset m(S)$ is left invariant if $f_{a} \in L$ for each $f \in L$. In this case $\varphi \in L^{*}$ is left invariant if $\varphi\left(f_{a}\right)=\varphi(f)$ for each $f$ in $L$ and $a$ in $S$. If $L$ contains the constant functions then $\varphi \in L^{*}$ is called a mean if $\varphi(f) \geqq 0$ for $f \geqq 0$ in $L$ and $\varphi\left(1_{S}\right)=1 . \varphi \in L^{*}$ is called a finite mean of $L^{*}$ if there is a finite subset $\left\{\alpha_{1}, \cdots, a_{n}\right\} \subset S$, and nonnegative $\alpha_{1}, \cdots, \alpha_{n}$ with $\Sigma \alpha_{i}=1$ such that $\varphi(f)=\sum_{i=1}^{n} \alpha_{i} f\left(\alpha_{i}\right)$ for each $f \in L$.

If $S$ is a topological semigroup then $J_{c} l(S)=\left\{\varphi \in C(S)^{*} ; \varphi\left(f_{a}\right)=\right.$ $\varphi(f)$ for each $f \in C(S)$ and $a \in S\}$ and $J l(S)=\left\{\varphi \in m(S)^{*} ; \varphi\left(f_{a}\right)=\varphi(f)\right.$ for each $f \in m(S)$ and $a \in S\}$. For "left-ideal group" or "compact leftideal group" see the introduction. A finite left ideal group is a left ideal group which contains a finite number of elements. If $X$ is a Banach space and $Y \subset X$ a subspace then we write $\operatorname{dim} Y=n$ if $Y$ is $n$ dimensional, $0 \leqq n<\infty$, and $\operatorname{dim} Y=\infty$ if $Y$ is not finite dimensional. 
If $X$ is a Banach space with conjugate space $X^{*}$ then the $w^{*}$ topology in $X^{*}$ (sometimes called the $X$ topology of $X^{*}$ ) is defined as in Day [5] p. 17.

A nonempty class $F$ of subsets of a set $S$ is called a field ( $\sigma$-field) if it is closed under complementation and under the operation of taking finite (countable) unions.

\section{The invariant mean on countable topological semigroups}

The main theorem of this chapter is Theorem 2. The main tool for its proof is Theorem 1. The proof of Theorem 1 uses basically the same idea as the proof of Theorem (5.1) of [6]. It yields though a simpler proof even for the discrete case than Theorem (5.1) of [6].

Definition 1. Let $S$ be a semigroup. Define $l_{a}: m(S) \rightarrow m(S)$ by $l_{a} f=f_{a}$ for any $a$ in $S$. If $L_{0} \subset m(S)$ is a left invariant manifold then define $l_{a}^{0}: L_{0} \rightarrow L_{0}$ by $l_{a}^{0} f=f_{a}$ for any $a$ in $S$ and $f$ in $L_{0}$. Denote in this case $\mathscr{L}_{a}=l_{a}^{*}: m(S)^{*} \rightarrow m(S)^{*}, \mathscr{L}_{a}^{0}=\left(l_{a}^{0}\right)^{*}: L_{0}^{*} \rightarrow L_{0}^{*}$ and

$$
J_{0} l(S)=\left\{\phi \in L_{0}^{*} ; \mathscr{L}_{s}^{0} \varphi=\varphi \text { for each } s \in S\right\} .
$$

THEOREM 1. Let $S$ be a left amenable semigroup and $L_{0} \subset m(S)$ be a left invariant linear manifold containing the constants. Assume that there is a sequence $\left\{s_{n}\right\}_{1}^{\infty} \subset S$ such that

$$
\left\{\varphi \in L_{0}^{*} ; \mathscr{L}_{s_{n}}^{0} \varphi=\varphi, n=1,2, \cdots\right\}=J_{0} l(S) .
$$

If $\operatorname{dim} J_{0} l(S)<\infty$ then each left invariant mean $\varphi \in L_{0}^{*}$ is a $w^{*-}$ sequential limit of finite means, in other words there is a sequence of finite means $\varphi_{n}$ in $L_{0}^{*}$ such that $\varphi(f)=\lim _{n \rightarrow \infty} \varphi_{n}(f)$ for each $f \in L_{0}$.

REMARK 1. If we do not assume the existence of a countable sequence $\left\{s_{n}\right\} \subset S$ as above then the theorem does not remain true as is shown by the following example: Let $G$ be an abelian compact hausdorff nonseparable topological group and let $L_{0}=C(G)$. Then $\operatorname{dim} J_{0} l(G)=1$. Let $\varphi_{0} \in L_{0}^{*}$ be the left invariant mean represented by the normalized Haar measure on $G$. Assume that $\varphi_{0}(f)=\lim _{n \rightarrow>\infty} \varphi_{n}(f)$ for each $f \in C(G)$ where $\varphi_{n}$ are finite means i.e., $\varphi_{n}(f)=\sum_{j=1}^{k} \alpha_{j} f\left(g_{j}\right)$ where $\alpha_{j}, g_{j}$ and $k$ depend on $n, \alpha_{j} \geqq 0$ and $\sum \alpha_{j}=1$.

If we call $\sigma\left(\varphi_{n}\right)=\left\{g_{1}, \cdots, g_{k}\right\}$, then $A=\bigcup_{n=1}^{\infty} \sigma\left(\varphi_{n}\right)$ is countable and therefore the group generated by $A$ is countable and therefore the closure of this group, say $G_{0}$, is a closed separable subgroup of $G$. Since $G$ is nonseparable $G \neq G_{0}$. But if $f \in C(G)$ satisfies $f(g) \geqq 1$ for $g \in G_{0}$ then $\varphi_{n}(f) \geqq 1$ since $\sigma\left(\varphi_{n}\right) \subset G_{0}$. Therefore $\varphi_{0}(f) \geqq 1$ which shows by [8] p. 248 that $\mu\left(G_{0}\right)=1$ where $\mu$ is the normalized Haar 
measure on $G$. But if $a \in G$ and $a \notin G_{0}$ then $a G_{0} \cap G_{0}=\varnothing$ and so $1=\mu(G) \geqq \mu\left(a G_{0}\right)+\mu\left(G_{0}\right)=2$, which is a contradiction.

Thus the above theorem is not true if we do not assume the existence of the above sequence $\left\{s_{n}\right\}$. This is the reason why Luthar, in his theorem about the uniqueness of the invariant mean on an abelian semigroup, see [11] and this author, in proving the theorem about the finite dimensionality of the set of invariant means on a semigroup, (see [6]), had to handle first the case in which the semigroup was countable and only afterwards, by using arguments involving much more the algebraic properties of semigroups, to handle the uncountable case (which is not yet proved in its due generality).

Proof of the Theorem. Let $\varphi_{0} \in L_{0}^{*}$ be a left invariant mean. Let $\psi \in m(S)^{*}$ be a norm preserving extension of $\varphi_{0}$. Since $1 \in L_{0}$ and $\varphi_{0}$ is a mean one has: $1=\left\|\varphi_{0}\right\|=\varphi_{0}(1)=\psi(1)$. But $\|\psi\|=\left\|\varphi_{0}\right\|$ and so $1=\|\psi\|=\psi(1)$. This implies as known that $\psi(f) \geqq 0$ if $f \geqq 0$. (In fact if $f \in m(S), 1 \geqq f \geqq 0$, would be such that $\psi(f)<0$ then $\|1-f\| \leqq 1$ and $\|\psi\| \geqq \psi(1-f)=\psi(1)-\psi(f)>1)$ and therefore $\psi$ is a mean. If $\nu$ is a left invariant mean on $m(S)$ then $\varphi_{0}^{\prime}=\nu \odot \psi$ is a left invariant mean on $m(S)$ (see Day [4] p. 526-527 and p. 529 Cor. 2) which is an extension of $\varphi_{0}$. In fact, if $f \in L_{0}$ then $(\nu \odot \psi)(f)=$ $\nu(h)$ where $h(s)=\psi\left(l_{s} f\right)=\varphi_{0}\left(l_{s} f\right)=\varphi_{0}(f)$. Thus $h(s)$ is constant on $S$ and takes only the value $\varphi_{0}(f)$. Hence $\nu(h)=\varphi_{0}(f)$, since $\nu$ is a mean. (We notice that we could have applied an invariant extension theorem of R. J. Silverman see [16] in order to get immediately the existence of $\varphi_{0}^{\prime}$ but we prefered the above simple argument).

Let now $\left\{\varphi_{a}^{\prime}\right\}$ be a net of finite means in $m(S)^{*}$ such that $w^{*}-\lim _{a} \varphi_{a}^{\prime}=$ $\varphi_{0}^{\prime}$ and $\lim _{\alpha}\left\|\mathscr{L}_{s} \varphi_{a}^{\prime}-\varphi_{a}^{\prime}\right\|=0$ for each $s$ in $S$. (see [6] p. 44, (5.8)*). If $\varphi_{a} \in L_{0}^{*}$ is the restriction of $\varphi_{a}^{\prime}$ to $L_{0}$ then since $\lim _{a} \varphi_{a}^{\prime}(f)=\varphi_{0}^{\prime}(f)$ for each $f \in m(S)$ we get that $\lim _{a} \varphi_{a}(f)=\varphi_{0}(f)$ for each $f \in L_{0}$ and thus $w^{*}-\lim \varphi_{a}=\varphi_{0}$ (in $L_{0}^{*}$ ). Moreover if $f \in L_{0}$ and $\|f\| \leqq 1$,

$$
\begin{aligned}
\left|\left(\mathscr{L}_{s}^{0} \varphi_{a}-\varphi_{a}\right) f\right| & =\left|\varphi_{a}\left(l_{s}^{0} f-f\right)\right|=\left|\varphi_{a}^{\prime}\left(l_{s} f-f\right)\right| \\
& =\left|\left(\mathscr{L}_{s} \varphi_{a}^{\prime}-\varphi_{a}^{\prime}\right) f\right| \leqq\left\|\mathscr{L}_{s} \varphi_{a}^{\prime}-\varphi_{a}^{\prime}\right\| \rightarrow 0
\end{aligned}
$$

for each $s$ in $S$. This implies that $\lim _{a}\left\|\mathscr{L}_{s}^{0} \varphi_{a}-\varphi_{a}\right\|=0$ (where the norm now is that of $L_{0}^{*}$ ) for each $s$ in $S$.

Let now $S\left(\varphi_{0}, 1 / n\right)=\left\{\phi \in L_{0}^{*} ;\left\|\varphi-\varphi_{0}\right\|<1 / n\right\}$ and let $V_{n}$ be a sequence of convex $w^{*}$ neighborhoods of $\varphi_{0}$ which are $w^{*}$-closed such that $V_{n+1} \subset V_{n}$ for $n=1,2, \cdots$ and

$$
\varphi_{0} \in V_{n} \cap J_{0} l(S) \subset S\left(\varphi_{0}, \frac{1}{n}\right) \cap J_{0} l(S) .
$$

The choice of such $V_{n}^{\prime}$ 's is possible since $J_{0} l(S)$ is finite dimensional 
(see [6] p. $44(5.5)^{*}$ and p. 45). There is now an $\alpha_{n}^{\prime}$ such that $\alpha \geqq \alpha_{n}^{\prime}$ implies $\left\|\mathscr{L}_{s_{i}}^{0} \varphi_{a}-\varphi_{a}\right\|<1 / n$ for $i=1,2, \cdots, n$.

Since $\varphi_{0}$ is a $w^{*}$ limit point of the net $\left\{\varphi_{a}\right\}$ there is an $\alpha_{n} \geqq \alpha_{n}^{\prime}$ such that $\varphi_{a_{n}} \in V_{n}$. Write $\varphi_{a_{n}}=\varphi_{n}$ and let $\psi_{0}$ be some $w^{*}$-limit point of the net $\left\{\varphi_{n}\right\}$. The set of means of $L_{0}^{*}$ can be written as

$$
\bigcap_{f \in L_{0}^{*} f \geqq 0}\left\{\varphi \in L_{0}^{*} ;\|\varphi\| \leqq 1 \text { and } \varphi(f) \geqq 0\right\}
$$

and so is $w^{*}$ compact. This shows the existence of such a $\psi_{0}$ (and so $\psi_{0}$ is even a mean). Moreover, if $f \in L_{0}\|f\| \leqq 1$ and $s_{j}$ is fixed then

$$
\begin{aligned}
\left|\left(\mathscr{L}_{s_{j}}^{0} \psi_{0}-\psi_{0}\right) f\right| \leqq & \left|\mathscr{S}_{s_{j}}^{0}\left(\psi_{0}-\varphi_{n}\right) f\right| \\
& +\left|\left(\mathscr{L}_{s_{j}}^{0} \varphi_{n}-\varphi_{n}\right) f\right|+\left|\left(\varphi_{n}-\psi_{0}\right) f\right| \\
\leqq & \left|\left(\psi_{0}-\varphi_{n}\right) l_{s_{j}}^{0} f\right| \\
& +|| \mathscr{L}_{s_{j}}^{0} \varphi_{n}-\varphi_{n}||+\left|\left(\varphi_{n}-\psi_{0}\right) f\right| .
\end{aligned}
$$

If $\varepsilon>0$ is given then there is an $n_{0} \geqq j$ such that $1 / n_{0}<\varepsilon / 3$ and therefore for $n \geqq n_{0},\left\|\mathscr{L}_{s_{j}}^{0} \varphi_{n}-\varphi_{n}\right\|<\varepsilon / 3$. Since $\psi_{0}$ is a $w^{*}$-limit point of $\left\{\varphi_{n}\right\}$, there is an $n_{1} \geqq n_{0}$ such that $\left|\left(\psi_{0}-\varphi_{n_{1}}\right) l_{s,}^{0} f\right|<\varepsilon / 3$ and $\left|\left(\varphi_{n}-\psi_{0}\right) f\right|<\varepsilon / 3$. Thus $\mathscr{L}_{s_{j}}^{0} \psi_{0}=\psi_{0}$ for each $j$ and using the assumption of our theorem we get that $\psi_{0} \in J_{0} l(S)$. But $\psi_{0}$ is also a $w^{*}$ limit point of the sequence $\left\{\varphi_{n}\right\}_{n=k}^{\infty} \subset V_{k}$. Since $V_{k}$ is $w^{*}$ closed $\psi_{0} \in V_{k}$ for each $k$. Thus $\psi_{0} \in V_{k} \cap J_{0} l(S) \subset S\left(\varphi_{0}, 1 / k\right) \cap J_{0} l(S)$. This shows that $\left\|\psi_{0}-\varphi_{0}\right\|<1 / k$ for each $k$ and so $\varphi_{0}=\psi_{0}$. Therefore the sequence $\left\{\varphi_{n}\right\} \subset L_{0}^{*}$ has the unique $w^{*}$-limit point $\varphi_{0}$. Therefore $\lim _{n \rightarrow>\infty} \varphi_{n}(f)=\varphi_{0}(f)$ for each $f \in L$ (see [6] p. 43 and replace there $m(G)$ by $L_{0}$ ). This finishes the proof of our theorem.

REMARK 2. $J_{0} l(S)$ coincides with the linear manifold spanned by the left invariant means in $J_{0} l(S)$. Since if $\varphi \in J_{0} l(S)$ and $\psi \in m(S)^{*}$ is any extension of $\varphi$ and if $\nu$ is any left invariant mean of $m(S)^{*}$ then $\varphi^{\prime}=\nu \odot \psi \in m(S)^{*}$ is a left invariant extension of $\phi \in J_{0} l(S)$ (see begining of proof of the preceeding theorem). But by [6] p. 55 footnote 5 there are left invariant means $\varphi_{1}^{\prime}, \varphi_{2}^{\prime}$ in $m(S)^{*}$ such that $\varphi^{\prime}=$ $\alpha \varphi_{1}^{\prime}-\beta \varphi_{2}^{\prime}$. If $\varphi_{i}$ is the restriction of $\varphi_{i}^{\prime}$ to $L_{0}$ then $\varphi=\alpha \varphi_{1}-\beta \varphi_{2}$ and $\varphi_{i}$ are left invariant means of $L_{0}^{*}$.

Definition 2. If $X$ is a topological space then $A \subset X$ is called a $Z$-set if $A=\{x ; f(x)=0\}$ for some $f \in C(X), F_{X}$ will denote the field generated by the $Z$-sets and $B_{X}$ is the $\sigma$-field generated by the $Z$-sets (or the $\sigma$-field of Baire subsets of $X$ ).

LEMMA 1. Let $S$ be a countable topological semigroup which is left amenable as a discrete semigroup. If the set of left invariant 
elements of $C(S), J_{c} l(S)$, is finite dimensional then each left invariant mean $\varphi_{0}$ of $C(S)^{*}$ can be represented by a regular countable additive measure $m_{0}$ on $B_{S}$.

Proof. Let $\varphi \in C(S)^{*}$ be a left invariant mean. Taking in the previous theorem $L_{0}=C(S)$ we get that there is a sequence of finite means $\left\{\varphi_{n}\right\}$ such that $\lim _{n \rightarrow>\infty} \varphi_{n}(f)=\varphi(f)$ for each $f \in C(S)$.

If $a \in S$ then let $m_{a}$ be the countable additive measure defined on $B_{S}$ by: $m_{a}(B)=1$ if and only if $a \in B . \quad m_{a}$ is regular and countably additive and since any finite mean can be represented by a linear combination of $m_{a}$ 's we get that $\varphi_{n}$ are represented by countable additive regular measures $m_{n}$ on $B_{S}$. Thus for each $f \in C(S)$

$$
\varphi_{0}(f)=\lim _{n \rightarrow \infty} \int f d m_{n} .
$$

Applying now A. D. Alexandroff's theorem (for statement and proof see Varadarajan [17] p. 68-69 Theorem 19) there exists a countably additive measure $m_{0}$ on $F_{S}$ such that

$$
\varphi_{0}(f)=\int f d m_{0} \text { for each } f \in C(S) .
$$

By a known theorem $m_{0}$ can be uniquely extended to a countably additive measure on $B_{S}$. (see [17] p. $45 \mathrm{Thm}$ 18). By the second part of [17] Thm. 18 p. 45 this $m_{0}$ is even regular.

REMARK 3. Applying now the uniqueness part of Alexandroff's theorem on the representation of linear functionals by measures, (see Alexandroff [1] or Varadarajan [17] p. $39 \mathrm{Thm}$ 5) we get that for any $Z$-set $Z_{0}$ one has $m_{0}\left(Z_{0}\right)=\inf \left\{\varphi_{0}(f) ; f \geqq 1_{Z_{0}}, f \in C(S)\right\}$.

THEOREM 2. Let $S$ be a countable topological semigroup which is left amenable as a discrete semigroup and which is a $T_{1}$ and regular topological space (for definition see [10] p.113). Then $\operatorname{dim} J_{c} l(S)=$ $n, n<\infty$, if and only if $S$ contains exactly $n$ finite left-ideal groups.

REMARK 4. (a) If $\varphi$ is any invariant mean on $m(S)$ then its restriction to $C(S)$ is an invariant mean of $C(S)^{*}$. Thus in any case $\operatorname{dim} J_{c} l(S) \geqq 1$ (if $S$ is left amenable as a discrete semigroup).

(b) Two different left-ideal groups are disjoint (each one is a minimal left ideal).

Proof of Theorem. $S$ being countable is Lindelöf and being also regular is normal (see Kelley [10] p. 113) We show now that any closed $F \subset S$ is a $Z$-set. 
Let $S-F=\left\{s_{1}, s_{2}, \cdots\right\}$ and let $f_{n} \in C(S)$ satisfy $0 \leqq f_{n} \leqq 1$ and $f_{n}(F)=0$ while $f_{n}\left(s_{n}\right)=1$ (Uryson's lemma).

Let $f(s)=\sum_{n=1}^{\infty}\left(1 / 2^{n}\right) f_{n}(s)$. Then $f \in C(S)$ and $\{s ; f(s)=0\}=\{F\}$. (This is the standard well known proof that any closed $G_{\delta}$ in a normal space is a $Z$-set).

Let $\varphi_{0}$ be a left invariant mean on $C(S)$ and let $m_{0}$ be the regular countably additive measure such that

$$
\varphi_{0}(f)=\int f d m_{0} \text { for each } f \in C(S) .
$$

If $S=\left\{t_{1}, t_{2}, \cdots\right\}$, then $1=m_{0}(S)=\sum_{n=1}^{\infty} m_{0}\left(\left\{t_{1}\right\}\right)$. Therefere there is some $a \in S$ (one of the $t_{i}$ 's) such that $m_{0}(\{a\})>0$. Now for any finite subset $F \subset S$

$$
\begin{aligned}
m_{0}(\{s F\}) & =\inf \left\{\varphi_{0}(f) ; f \geqq 1_{s F}\right\}=\inf \left\{\varphi_{0}\left(f_{s}\right) ; f \geqq 1_{s F}\right\} \\
& \geqq \inf \left\{\varphi_{0}(h) ; h \geqq 1_{F}\right\}=m_{0}(F) .
\end{aligned}
$$

And the inequality is true since $f \geqq 1_{s F}$ implies that $f_{s}(t)=f(s t) \geqq 1$ for $t \in F$ i.e. $f_{s} \geqq 1_{F}$.

Therefore if $a \in S$ satisfies $m_{0}(\{a\})>0$ and $s \in S$ we have $m_{0}(\{s a\}) \geqq$ $m_{0}(\{a\})>0$. This shows that $S a$ is a finite left ideal (since $m_{0}(S)=1$ ). If $A \subset S a$ is a minimal left ideal then for $b \in A, A b \subset A$ and since $A b$ is a left ideal, $A b=A$. If we denote $A=\left\{b_{1}, \cdots, b_{N}\right\}$, the above shows that for each pair $i, j, 1 \leqq i, j \leqq N$, there is some $k, 1 \leqq k \leqq N$, such that $b_{k} b_{i}=b_{j}$. Taking $F=\left\{b_{i}\right\}$ in the inequality (1) we get that $m_{0}\left(\left\{b_{j}\right\}\right)=m_{0}\left(\left\{b_{k} b_{i}\right\}\right) \geqq m_{0}\left(\left\{b_{i}\right\}\right)>0$ and interchanging $i$ and $j$ we get that $m_{0}\left(\left\{b_{j}\right\}\right)=m_{0}\left(\left\{b_{i}\right\}\right)>0$ for each $b_{i}, b_{j}$ in the finite minimal left ideal $A$, i.e. $m_{0}\left(\left\{b_{1}\right\}\right)=m_{0}\left(\left\{b_{2}\right\}\right)=\cdots=m_{0}\left(\left\{b_{N}\right\}\right)$. If now $b$ is any element of $A$ then $m_{0}(b A) \geqq m_{0}(A)=N m_{0}\left(\left\{b b_{1}\right\}\right)$. But $b A \subset A$ and therefore $m_{0}(b A)=$ $j m_{0}\left(\left\{b b_{1}\right\}\right)$ where $j$ is the number of differents elements in $b A$. Thus $j=N$ and $b A=A$. This shows that $A$ is a finite minimal left ideal which satisfies for each $b \in A$ that $b A=A b=A$. This shows that $A$ is a finite left ideal group.

If $s \in S$ and $e$ is the identity of $A$ then $s A=(s e) A=A$ since $s e \in A$. Thus $s A=A$ so that any finite left-ideal group is also what is (unnecessarily) called in [6] p. 34 a (l.i.l.c). (Also, obviously, any finite group and (l.i.l.c) is a left-ideal group.) Now the number of finite left-ideal groups in $S$ is less than or equal to $n$ (where $\operatorname{dim} J_{c} l(S)=n$ ) since if $A_{1}, \cdots, A_{n}, A_{n+1}$ would be finite left-ideal groups and we would define $\varphi_{i} \in C(S)$ by $\varphi_{i}(f)=\left[1 / N\left(A_{i}\right)\right] \sum_{s \in A_{i}} f(s)$ where $N\left(A_{i}\right)$ is the number of elements of $A_{i}$ then as easily checked $\varphi_{i}$ is a left invariant mean on $C(S)$ (since $s A_{i}=A_{i}$ for each $s \in S$ ). But $\varphi_{1}, \cdots, \varphi_{n+1} \in C(S)^{*}$ are linearly independent. In fact if $\sum_{1}^{n+1} \alpha_{i} \varphi_{i}=0$ and if we define $f_{i}^{\prime}$ on $\mathrm{U}_{1}^{n+1} A_{j}$ by $f_{i}^{\prime}(s)=1$ for $s \in A_{i}$ and $f_{i}^{\prime}(s)=0$ if $s \in A_{j}$ for $j \neq i$ then 
we can, by Tietze's extension theorem find an extension $f_{i} \in C(S)$ of $f_{i}^{\prime}$. For this $f_{i}$ we have $0=\sum \alpha_{j} \varphi_{j}\left(f_{i}\right)=\alpha_{i} \varphi_{i}\left(f_{i}\right)=\alpha_{i}$ which shows that $\alpha_{i}=0$ so that $\operatorname{dim} J_{c} l(G) \geqq n+1$, which contradicts our assumption. Thus there are at most $n$ finite left-ideal groups in $S . *$ If $m(1 \leqq m \leqq n)$ is the number of the finite left-ideal groups in $S$ then we get by [6] p. 34 Thm. 3.1 and p. 36 Remark 3.2 and [6] p. 55 footnote 5 that $\operatorname{dim} J l(S)=m$ where $J l(S)$ is the set of left invariant elements of $\mathrm{m}(S)^{*}$. But any $\varphi \in J_{\mathrm{c}} l(S) \subset C(S)^{*}$ has an extention $\varphi^{\prime} \in J l(S) \subset m(S)^{*}$ (see beginning of proof to Thm (II.1)). Thus if $\varphi_{1}, \cdots, \varphi_{n}$ are $n$ linearly independent elements of $J_{c} l(S)$ and $\left\{\varphi_{1}^{\prime}, \cdots, \varphi_{n}^{\prime}\right\} \subset J l(S) \subset m(S)^{*}$ are extensions of $\varphi_{1}, \cdots \varphi_{n}$ respectively then $\varphi_{1}^{\prime}, \cdots, \varphi_{n}^{\prime}$ are also linearly independent in $m(S)^{*}$. Since if $\sum_{1}^{n} \alpha_{i} \varphi_{1}^{\prime}=0$ then for each $f \in C(S) \subset m(S)$ we would have $\sum_{1}^{n} \alpha_{i} \varphi_{i}(f)=0$ which would imply that $\alpha_{1}=\alpha_{2}=\cdots \alpha_{n}=0$. Therefore $\left\{\varphi_{1}^{\prime}, \cdots, \varphi_{n}^{\prime}\right\} \subset J l(S)$ are linearly independent which shows that $m \geqq n$ and $S$ contains exactly $n$ finite left-ideal groups.

REMARK 5. We also proved at the end of this theorem that $\operatorname{dim} J_{c} l(S)=n$ implies $\operatorname{dim} J l(S)=n$ where $S$ is countable and left amenable as a discrete semigroup. That this does not hold true for noncountable $S$ is shown by the following example: Let $G$ be an abelian compact Hausdorff topological group which is not finite. Then by Theorem B of [6] p. 32 we get that $\operatorname{dim} J l(G)=\infty$ while $\operatorname{dim} J_{c} l(G)=1$ (The Haar measure is unique). In other words the restriction of the infinite dimensional space $J l(G) \subset m(G)^{*}$ to $C(G)$ forms an one dimensional subspace of $C(G)^{*}$ which coincides with $J_{c} l(G)$. The end of the proof of our preceeding theorem shows that this cannot happen if $G$ is countable.

CoRollary 1. Let $S$ be a countable $T_{1}$ regular topological semigroup which is left amenable as a discrete semigroup. If $S$ has left cancellation then $\operatorname{dim} J_{c} l(G)=n(n<\infty)$ if and only if $S$ is finite and is the union of $n$ finite disjoint left-ideal groups. $\operatorname{dim} J_{c} l(G)=1$ if and only if then $G$ is a finite group.

Proof. At the end of the last theorem it was in fact shown that $n=\operatorname{dim} J_{c} l(S)=\operatorname{dim} J l(S)$ where $J l(S)$ is the set of left invariant

* We could also proceed as follows: Let $m, 1 \leqq m<\infty$, be the number of finite left ideal groups of $S$ and let $A$ be a compact left ideal group of $S$. Then $A$ is a countable group and has a compact hausdorff topology in which multiplication is separately continuous. Hence by the theorem of Ellis (see Ellis [21] or GlicksbergDeleeuw [19] p.p. 64-65 and p.p. 94-96) $A$ is a compact topological group which is countable. Hence $A$ has to be finite (since if $m$ is its normalised Haar measure then $m\{\alpha\}>0$ for some $a$ in $A$, hence $m(A)=\infty$, if $A$ is infinite, which cannot be.). Therefore $S$ contains, in our case exactly $m$ compact left ideal groups. By Theorem IV -1 of the present paper $\operatorname{dim} J_{o} 1(S)=m$ which finishes the proof. 
elements of $m(S)^{*}$. Applying Thm $E$ of [6] p. 49 and remembering footnote 5 on p. 55 of [6] we get this corollary.

REMARK 6. If $G$ is a discrete amenable group and $G^{\prime} \subset G$ a subgroup then there exists a linear positive isometry from $J l\left(G^{\prime}\right)$ into $J l(G)$ (see Day [4] p. 534). Therefore, the assumption that $\operatorname{dim} J l(G)=n$ implies that $\operatorname{dim} J l\left(G^{\prime}\right) \leqq n$. If $G$ is a topological group and $G^{\prime} \subset G$ a subgroup then there does not generally exist a linear isometry from $J_{c} l\left(G^{\prime}\right)$ to $J_{c} l(G)$. In fact let $G$ be a compact abelian hausdorff topological group. Then $\operatorname{dim} J_{c} l(G)=1$. If now $G^{\prime} \subset G$ is any countable (not finite) subgroup then $G^{\prime}$ being abelian, is amenable as a discrete group and satisfies all the assumptions of our previous corollary. Therefore $\operatorname{dim} J_{c} l\left(G^{\prime}\right)=\infty$, which shows that there cannot exist an isometry from $J_{c} l\left(G^{\prime}\right)$ into $J_{c} l(G)$. This theorem of Day was the main tool to pass from the countable case to the uncountable case when dealing with discrete groups (see [6] p. 46 proof of Cor (5.3)). The above example shows that this important tool is not more available when dealing with topological groups.

\section{The invariant mean on separable topological groups}

The main theorem of this chapter is Theorem 1 . We have to restrict ourselves to topological groups rather than topological semigroups since our method works only for left uniformly continuous functions and on semigroups there may not be any uniformity at all which is consistent with the algebraic structure.

DeFINITION 1. Let $G$ be a topological group and $U \subset G$ a neighborhood of the identity. We say that $U$ totally covers $G$ if $G \subset \bigcup_{i=1}^{k} U a_{i}$ : for some finite subset $\left\{a_{1}, \cdots, a_{k}\right\} \subset G$. (We should have said that $U$ left totally covers $G$ but we drop the "left" since we do not deal at all with the "right" case.)

We say that the topological group $G$ has property (B) if it has a neighborhood of the identity $U$ such that none of its powers totally covers $G$ (or in other words for each $n$ and each finite subset $\left\{a_{1}, \cdots, a_{k}\right\} \subset G, G-\bigcup_{i=1}^{k} U^{n} a_{i} \neq \varnothing$.)

REMARK 1.

(a) A noncompact locally compact group has property $B$ since if $U$ is a compact neighborhood of the identity then $U^{n}$ is compact for each $n$ and so $\bigcup_{i=1}^{k} U^{n} a_{i}$ is compact and therefore does not cover the whole of $G$.

(b) Any subgroup $G \neq\{0\}$ of a hausdorff locally convex linear topological space $E$ has property $B$. Since if $0 \neq a \in G$ and $f$ is a 
linear continuous functional on $E$ such that $f(a) \neq 0$ then let $U=$ $\{y \in G ;|f(y)|<1\}$. Then the $n^{\text {th }}$ power of $U$ is defined by $U^{n}=$ $U+\cdots+U$ ( $n$ times). Thus if $y \in U^{n}$ then $y=u_{1}+\cdots+u_{n}$ with $u_{i} \in U$ and $|f(y)| \leqq n$. Therefore $y \in \bigcup_{i=1}^{k} U^{n}+g_{i}$ implies that

$$
|f(y)| \leqq \max _{1 \leqq i \leqq k}\left|f\left(g_{i}\right)\right|+n=K \text {. }
$$

But there is a positive integer $j$ which satisfies $|f(j a)|=j .|f(a)|>K$. Since $j a \in G$ this implies that $G$ is not included in $U\left(g_{i}+U^{n}\right)$ so that $G$ has property (B).

(C) Any topological metric group $G$ which admits a right invariant non bounded metric (i.e. its topology can be given by a right invariant metric $d$ such that for any $K>0$ there are $a, b \in G$ satisfying $d(a, b)>K)$, has property $B$. It should be pointed out that any metric topological group admits as known (see G. Birkhof [2] or Kakutani [9]) a right invariant metric. Therefore the real requirement is that the metric should be unbounded. (If $G$ is totally bounded and metric then any admissible invariant metric is bounded).

Assume that $G$ admits a right invariant unbounded metric $d$. If $e$ is the identity element of $G$ then let $U=\{g ; d(e, g)<1\}$. Then for $u \in U^{n} d(e, u) \leqq n$. This is true for $n=1$. Assume that it holds for $n-1$. If $u \in U^{n}$ then $u=u_{1} u_{2} \cdots u_{n}$ with $u_{i} \in U$. Then

$$
\begin{aligned}
d\left(u_{1} u_{2} \cdots u_{n}, e\right) & \leqq d\left(u_{1} u_{2} \cdots u_{n}, u_{2} \cdots u_{n}\right)+d\left(u_{2} \cdots u_{n}, e\right) \\
& \leqq d\left(u_{1}, e\right)+n-1 \leqq n
\end{aligned}
$$

since $d$ is right invariant. If $G \subset \bigcup_{i=1}^{k} U^{n} a_{i}$ then any $g \in G$ satisfies $g \in U^{n} a_{i}$ for some $1 \leqq i \leqq k$ and so $g=v a_{i}$ with $v \in U^{n}$. Thus

$$
d(e, g) \leqq d\left(e, a_{i}\right)+d\left(a_{i}, v a_{i}\right) \leqq K+d(e, v) \leqq K+n=K_{1}
$$

where $K=\max \left\{d\left(e, a_{i}\right), 1 \leqq i \leqq k\right\}$. But the metric $d$ is unbounded and therefore there are $a, b \in G$ such that $d\left(e, b a^{-1}\right)=d(a, b)>K_{1}$ which is a contradiction. (As we see here it is enough that $d(x, y)$ should be a continuous unbounded right invariant pseudometric on $G$ and it is not necessary that $d$, generates the topology of $G)^{4}$

The following lemma is needed in what follows:

LEMMA 1. Let $G$ be a separable hausdorff topological group having property (B) and let $\left\{p_{j}\right\}_{1}^{\infty}$ be dense in $G$. Then for any open sym-

4 The following example of a group with property (B) seems to have some interest. Consider the space $L_{p}(0,1)$, for $0<p<1$, with the metric $\int_{0}^{1}|x(t)-y(t)| p d t$. The function $F(x)=\int_{0}^{1}|x(t)|^{p} d t$ is uniformly continuous and $F(n x)=n^{p} \int_{0}^{1}|x(t)|^{p} d t \rightarrow \infty$ if $n \rightarrow \infty$ and $x \neq 0$. As known there is no nonzero continuous linear functional (or even character) on $L_{p}(0,1)$, for $0<p<1$, and hence it is not even a locally convex linear topological space (see M. M. Day Bull. Amer. Math. Soc. 46 (1940), 816-823). 
metric neighborhood of the identity $U$ none of whose powers totally cover $G$ there exist a left uniformly continuous nonnegative function $F$ on $G$ such that

$$
\{g ; 0 \leqq F(g) \leqq k\}=F^{-1}([0, k]) \subset \bigcup_{j=1}^{k+2} U_{p_{j}}^{2(k+2)} \quad \text { for } k=1,2,3, \cdots
$$

Proof. Let $\left\{p_{n}\right\}$ be a countable dense subset of $G$. We define an increasing sequence of open subsets of $G$ in the following way:

$$
A_{1}=U p_{1} \text {. }
$$

As well known $\bar{A}_{1}=\cap V A_{1}$ where $V$ ranges over all the neighborhoods of $e$ and therefore $\bar{A}_{1} \subset U^{2} p_{1}$. Let

$$
A_{2}=U\left(\bar{A}_{1} \cup U p_{2}\right) \text {. }
$$

We get immediately that $U p_{1} \cup U p_{2} \subset A_{2}$ and $U \bar{A}_{1} \subset A_{2}$ and

$$
\begin{aligned}
& \bar{A}_{2} \subset U\left[U\left(\bar{A}_{1} \cup U p_{2}\right)\right] \\
& \quad \subset U^{2}\left(U^{2} p_{1} \cup U p_{2}\right) \subset U^{4} p_{1} \cup U^{3} p_{2} \subset U^{4} p_{1} \cup U^{4} p_{2} .
\end{aligned}
$$

Assume now that $A_{1}, A_{2}, \cdots, A_{n}$ have been chosen such that

$$
U p_{1} \cup U p_{2} \cdots \cup U p_{j} \subset A_{j} \subset \bar{A}_{j} \subset U^{2 j} p_{1} \cup U^{2 j} p_{2} \cdots \cup U^{2 j} p_{j}
$$

and $U \bar{A}_{j-1} \subset A_{j}$ for each $j \leqq n-1$ then we chose

$$
A_{n}=U\left(\bar{A}_{n-1} \cup U p_{n}\right) \text {. }
$$

We have that $\left(U p_{1} \cup U p_{2} \cdots \cup U p_{n}\right) \subset\left(A_{n-1} \cup U p_{n}\right) \subset A_{n}$ and that

$$
\begin{aligned}
U \bar{A}_{n-1} & \subset A_{n} \subset \bar{A}_{n} \subset U^{2}\left(\bar{A}_{n-1} \cup U p_{n}\right) \\
& \subset U^{2}\left[U^{2(n-1)} p_{1} \cup \cdots \cup U^{2(n-1)} p_{n-1} \cup U^{2(n-1)} p_{n}\right] \\
& \subset U^{2 n} p_{1} \cup \cdots \cup U^{2 n} p_{n} .
\end{aligned}
$$

In short our sequence of open substs $A_{n}$ satisfies the following

$$
\bigcup_{n=1}^{\infty} A_{n}=G
$$

$\left(\bigcup_{i=1}^{\infty} U p_{i} \subset \bigcup_{n=1}^{\infty} A_{n}\right.$ and $G=\bigcup_{i=1}^{\infty} U p_{i}$ since otherwise there would be some $a \in G$ such that $a \notin U p_{i}$ for each $i$ i.e., $p_{i} \notin U \alpha$ for each $i$ ( $U$ is open symetric) which cannot be since $\left\{p_{i}\right\}$ is dense in $G$.)

$$
U \bar{A}_{n} \subset A_{n+1} \subset \bigcup_{i=1}^{n+1} U^{2(n+1)} p_{i} .
$$

We can also assume that $A_{n}-A_{n-1} \neq 0$ for each $n$ (Where for $A, B \subset G$, $A-B$ are the elements of $A$ which are not in $B$ ). (Since otherwise we would choose $A_{n_{1}}=A_{1}, n_{2}$ to be the first $n>n_{1}$ for which $A_{n}-A_{n_{1}}=\varnothing$ and if $n_{k-1}$ was already chosen then let $n_{k}$ be the first $n>n_{k-1}$ for 
which $A_{n}-A_{n_{k-1}} \neq \varnothing$. There is such a $n_{k}$ since $U^{k}$ does not totally cover $G$ for any $k$. Obviously the sequence $A_{k}^{\prime}=A_{n_{k}}$ would satisfy (III. 1) (III. 2) in addition to $A_{k}^{\prime}-A_{k-1}^{\prime} \neq \varnothing$ )

It is proved in A. Weil [18] p. 13 that if $E$ is a uniform space and $V^{\prime}$ a neighborhood of the diagonal in $E \times E$ and if $p_{0} \in E$ then there exists a uniformly continuous function $f: E \rightarrow[0,1]$ such that $f(p)=0$ and $f(q)=1$ for $q \in E-V^{\prime}(p)$. (where $V^{\prime}(p)=\left\{q \in E ;(p, q) \in V^{\prime}\right\}$ and for $\left.A \subset E, V_{\alpha}^{\prime}(A)=\bigcup_{p \in A} V_{\alpha}^{\prime}(p)\right)$. But moreover, if we chose a fixed sequence of symmetric neighborhoods of the diagonal (i.e., elements of the uniformity) in $E \times E$ say $V_{n}^{\prime}$ which satisfy $V_{n+1}^{\prime} V_{n+1}^{\prime} \subset V_{n}^{\prime}$ (for notation see [18] A. Weil) for $n=0,1,2 \cdots$ and $V_{0}^{\prime} \subset V^{\prime}$ then the function $f: E \rightarrow[0,1]$ can even be chosen to satisfy the condition $|f(q)-f(r)|<1 / 2^{m-1}$ whenever $(p, q) \in V_{m}^{\prime}$ (see [18] p. 14). We notice also that the sequence $V_{n}^{\prime}$ is not dependent upon $p$. But the same proof yields actually more: If $V^{\prime}$ is a neighborhood of the diagonal (a member of the uniformity) and the sequence $V_{n}^{\prime}$ is chosen as above and if $P$ is any subset of $E$ then there exists a uniformly continuous $f: E \rightarrow[0,1]$ such that $f(p)=0$ if $p \in P$ and $f(q)=1$ if $q \in E-V^{\prime}(P)$. Returning now to our group we consider its left uniformity i.e. the uniformity whose elements are all the sets of the form $V^{\prime}=\{(p, q) ; q \in V p\}$ where $p, q \in G$ and $V$ ranges over all the neighborhoods of $e$. Let $V_{n}, n=0,1,2 \cdots$ be a fixed sequence of symmetric neighborhoods of $e$ in $G$ such that $V_{0}=U$ and $V_{n+1} V_{n+1} \subset V_{n}$ for each $n$. Then $V_{n}^{\prime}=$ $\left\{(p, q) ; q \in V_{n} p\right\} \subset G \times G$ are symmetric elements of the uniformity which satisfy $V_{n+1}^{\prime} V_{n+1}^{\prime} \subset V_{n}^{\prime}$ (since for each $p \in G \quad\left(V_{n+1}^{\prime} V_{n+1}^{\prime}\right)(p)=$ $\left.V_{n+1}^{\prime}\left(V_{n+1} p\right) \subset V_{n} p=V_{n}^{\prime}(p)\right)$. Therefore since $V_{0}^{\prime}\left(\bar{A}_{k}\right)=V_{0} \bar{A}_{k}=U \bar{A}_{k} \subset A_{k+1}$ there exists a left uniformly continuous function $f_{k}: G \rightarrow[0,1]$ such that $f_{k}\left(A_{k}\right)=0$ and $f_{k}\left(G-U \bar{A}_{k}\right)=1$ which implies that $f_{k}\left(G-A_{k+1}\right)=1$. Moreover if $(p, q) \in V_{m}^{\prime}$ i.e. if $q \in V_{m} p$ then $\left|f_{k}(p)-f_{k}(q)\right|<1 / 2^{m-1}$ for each $k$.

Consider now the sequence of functions

$$
h_{k}(g)=f_{k}(g)+k-1 \quad \text { for } k=1,2,3, \cdots
$$

We have:

$$
\begin{aligned}
& h_{1}(g)=\left\{\begin{array}{ll}
0 & \text { on } \bar{A}_{1} \\
1 & \text { on } G-A_{2}
\end{array} ;\right. \\
& h_{2}(g)= \begin{cases}1 \text { on } \overline{A_{2}} \\
2 \text { on } G-A_{3}\end{cases} \\
& h_{k}(g)=\left\{\begin{array}{cc}
k-1 \text { on } \bar{A}_{k} \\
k & \text { on } G-A_{k+1}
\end{array}\right.
\end{aligned}
$$

and also

(III. 3)

$$
\left|h_{k}(p)-h_{k}(q)\right|=\left|f_{k}(p)-f_{k}(q)\right|<1 / 2^{m-1}
$$


$q \in V_{m} P$ uniformly in $k$. (Our sequence of symmetric neighborhoods $V_{m}$ is the same for all $A_{k}$ ).

Define now the required function $F$ on $G$ as follows:

(III. 4)

$$
F(g)= \begin{cases}h_{1}(g) & \text { for } g \in A_{2} \\ h_{2}(g) & \text { for } g \in A_{3}-A_{2} \\ h_{k}(g) & \text { for } g \in A_{k+1}-A_{k} \text { if } k \geqq 2 .\end{cases}
$$

Since $A_{k} \subset A_{k+1}$ and $\mathrm{U}_{n=1} A_{k}=G, F$ is a well defined and real valued function on $G$ which satisfies that $\{g: 0 \leqq F(g) \leqq k\} \subset A_{k+2}$, since if $g \notin A_{k+2}$ then $g \in A_{n}-A_{n-1}$ for some $n>k+2$ and so

$$
F(g)=h_{n}(g) \geqq n-1 \geqq k+1 .
$$

Therefore by (III. 2) $F^{-1}([\mathrm{Ok}]) \subset \bigcup_{i=1}^{k+2} U^{2(k+2)} p_{i}$. We also notice that $F(g)$ is not bounded since $A_{k}-A_{k-1} \neq \varnothing$ and for $g \in A_{k}-A_{k-1}, F(g)=$ $h_{k}(g) \geqq k-1$. We prove now that $F$ is left uniformly continuous:

If $\varepsilon>0$ is given then there exists an $m$ such that $2^{-m+2}<\varepsilon$. We shall show that for any $p, q \in G$ such that $q \in V_{m} p,|F(p)-F(q)|<\varepsilon$. Assume therefore that $q \in V_{m} p$. If $p$ and $q$ are both in $A_{k+1}-A_{k}$ for some $k \geqq 2$ or are both in $A_{2}$ we can immediately conclude from (III. 3 ), (III. 4) that: $|F(p)-F(q)|=\left|h_{k}(p)-h_{k}(q)\right|<1 / 2^{m-1}<\varepsilon$ where $k=1$ if $p$ and $q$ are both in $A_{2}$.

If the above is not the case then let $i$ be the first index for which $p \in A_{i}$ and $j$ be the first index for which $q \in A_{j}$. Assume that $i<j$. Since $q \in V_{m} p \subset U p \subset U A_{i} \subset A_{i+1}$ (see (III. 2)) we have that $j=i+1$ and $q \in A_{i+1}$ (we can assume that $i \geqq 2$ since if $i=1$ then $p, q \in A_{2}$ and we alredy dealt with this case). Thus $p \in A_{i}-A_{i-1}$ and

$$
q \in A_{i+1}-A_{i} \subset G-A_{i} \text { and } q \in V_{m} p .
$$

Therefore:

$$
\begin{aligned}
|F(q)-F(p)| & =h_{i}(q)-h_{i-1}(p) \\
& =h_{i}(q)-(i-1)+(i-1)-h_{i-1}(q) \\
& =h_{i}(q)-h_{i}(p)+h_{i-1}(q)-h_{i-1}(p)
\end{aligned}
$$

since:

$$
h_{i-1}(g)=\left\{\begin{array}{l}
i-2 \text { on } \bar{A}_{i-1} \\
i-1 \text { on } G-A_{i}
\end{array}\right.
$$

and

$$
h_{i}(g)=\left\{\begin{array}{cc}
i-1 & \text { on } \bar{A}_{i} \\
i & \text { on } G-A_{i+1} .
\end{array}\right.
$$

Therefore, remembering that $q \in V_{m} p$ and applying (III. 4), we get:

$$
\begin{aligned}
F(q)-F(p) \mid & \leqq\left|h_{i}(q)-h_{i}(p)\right|+\left|h_{i-1}(q)-h_{i-1}(p)\right| \\
& \leqq 1 / 2^{m-1}+1 / 2^{m-1}<\varepsilon .
\end{aligned}
$$


If $j<i$ then, remembering that $q \in V_{m} p$ if and only if $p \in V_{m} q\left(V_{m}\right.$ is symmetric), and interchanging $p$ and $q$ we get that $|F(p)-F(q)|<\varepsilon$ for this case also.

Corollary. A topological group $G$ has property $B$ if and only if there exists a left uniformly continuous real valued unbounded function on $G^{5}$.

Proof. If $G$ has property B then the function $F(g)$ of the preceeding lemma is unbounded and left uniformly continuous.

Conversely if $F(g)$ is an unbounded left uniformly continuous function on $G$ there is a neighborhood of the identity $u$ such that $|F(a)-F(b)|<1$ if $b \in U a$ for any $a, b \in G$. We show now that if $b \in U^{n} a$ then $|F(a)-F(b)| \leqq n$. Assume that this is true for $n$. If $b \in U^{n+1} a$ then $b \in U\left(U^{n} a\right)$ and so there is some $c \in U^{n} a$ such that $b \in U c$. Therefore $|F(b)-F(c)|<1$ and so

$$
|F(b)-F(a)| \leqq|F(b)-F(c)|+|F(c)-F(a)| \leqq 1+n .
$$

Assume now now that $G=\bigcup_{i=1}^{k} U^{n} a_{i}$ where $a_{i} \in G$. If now $g \in G$ then $g \in U^{n} a_{i}$ for some $1 \leqq i \leqq k$ and so $\left|F(g)-F\left(a_{i}\right)\right| \leqq n$ which implies that

$$
|F(g)| \leqq n+\max _{1 \leqq i \leqq k}\left|F\left(a_{i}\right)\right|
$$

This contradicts the assumption that $F(g)$ is not bounded.

Definition 2. We denote by $L U C(G) \subset C(G)$ the norm closed subspace of $C(G)$ of left uniformly continuous functions on $G$, i.e. $f \in C(G)$ is in $\operatorname{LUC}(G)$ if and only if for each $\varepsilon>0$ there is a neighborhood of the identity, $V$ in $G$ such that $|f(v g)-f(g)|<\varepsilon$ for each $v \in V$ and $g \in G$.

5 This corollary is an immediate consequence of a theorem of M. Atsuji (see Canad. J. Math. 13 (1961), p. 661) who proved that it holds true for any uniform space. Thanks are due to $\mathrm{K}$. Ross and W. Comfort for communicating it to me. The above corollary (which is not used in what follows) gives in fact a characterization of what may be called "uniformly pseudocompact groups" i.e. groups for which every uniformly continuous real function is bounded. It states: Each left uniformly continuous real function on the topological group $G$ is bounded if and only if each neighborhood of the identity has some power which totally covers $G$ (see def. 1 of this ch.). The following example of an abelian metric group for which every uniformly continuous real function is bounded but the group is not totally bounded (i.e. its completion is not compact) has been given by W. W. Comfort and K. A. Ross in [23]. Let $G=T^{\aleph_{0}}$ (where $T$ is the circle group) and define for $x=\left\{x_{n}\right\}, y=\left\{y_{n}\right\}$ in $G, x \cdot y=\left\{x_{n} y_{n}\right\}$. The metric $d$ is defined by $d(x, y)=$ $\sup \left\{\left|x_{n}-y_{n}\right| ; n=1,2, \cdots\right\}$. These remarks and the above corollary are given here only for the general information of the reader and are not used later on. The lemma preceeding the above corollary is though, used heavily in what follows. 
$J_{u} l(G)$ will denote the space of left invariant element of $L U C(G)^{*}$. Since $L U C(G)$ will play the role of $L_{0}$ of Theorem II-1, define $l_{a}^{0} f=f_{a}$ for $a$ in $S$ and $f$ in $L U C(G)$. Also $\mathscr{L}_{a}^{0}=\left(l_{a}^{0}\right)^{*}$.

THEOREM 1. Set $G$ be a separable hausdorff topological group which is amenable as discrete group and satisfies property (B). Then $J_{u} l(G)$ is infinite dimensional. As an immediate consequence $J_{c} l(G)$ is infinite dimensional.

Proof. We remark first that $L U C(G)$ is a left invariant subspace of $m(G)$ containing the constant functions, since if $f \in L U C(G)$ and $a \in G$ then let $U$ be a neighborhood of the identity $e$ of $G$ such that $|f(u g)-f(g)|<\varepsilon$ for each $u$ in $U$ and $g$ in $G$. Then $|f(u a g)-f(a g)|<\varepsilon$ for $u$ in $U$ and $g$ in $G$. If $V$ is a neighborhood of $e$ such that $a V \subset U a$ then

$$
\left|\left(l_{a} f\right)(v g)-\left(l_{a} f\right)(g)\right|=|f(a v g)-f(a g)|<\varepsilon
$$

for each $v$ in $V$ and $g$ in $G$ which shows that $l_{a} f \in L U C(G)$.

$G$ is amenable as a discrete group and therefore there exists a left invariant mean $\mu$ on $m(G)$. The restriction of $\mu$ to $L U C(G)$ is a left invariant mean. Therefore in any case $\operatorname{dim} J_{u} l(G) \geqq 1$. Assume now that $\operatorname{dim} J_{u} l(G)=n$ where $0<n<\infty$. We shall show that in this case $G$ has not property (B). Let $\left\{p_{n}\right\}$ be a countable dense subset of $G$ and let $\varphi \in L U C(G)^{*}$ satisfy $\|\varphi\|=1$ and $\mathscr{L}_{p_{n}}^{0} \varphi=\varphi$ for $n=1,2, \cdots$. Let $a \in G$, then for $f \in L U C(G), \varphi\left(l_{p_{n}}^{0} f\right)=\varphi f$ and so:

$$
\begin{aligned}
\left(\mathscr{L}_{a}^{0} \varphi-\varphi\right) f & =\varphi\left(l_{a}^{0} f-f\right) \\
& =\varphi\left[\left(l_{a}^{0}-l_{p_{n}}^{0}\right) f\right]+\varphi\left(l_{p_{n}}^{0} f-f\right) \\
& =\varphi\left[\left(l_{a}^{0}-l_{p_{n}}^{0}\right) f\right] .
\end{aligned}
$$

But for any $\varepsilon>0$ there is a neighborhood $V$ of $e$ such that $|f(v g)-f(g)|<\varepsilon$ for $g$ in $G$ and $v \in V$, i.e. surely $|f(v a g)-f(a g)|<\varepsilon$ whenever $v \in V$. Thus for any $b \in V a$ we have that $\left\|\left(l_{b}^{0}-l_{a}^{0}\right) f\right\|<\varepsilon$. Since $p_{n}$ is dense in $G$ there is some $p_{j}$ in $V a$. For this $p_{j}$ we can write $\left|\left(\mathscr{L}_{a}^{0} \varphi-\varphi\right) f\right| \leqq\left|\varphi\left(l_{a}^{0}-l_{p}^{0}\right)(f)\right| \leqq\left\|\left(l_{a}^{0}-l_{p_{n}}^{0}\right) f\right\|<\varepsilon$. This shows that $\varphi \in J_{u} l(G)$ or that $\left\{\varphi \in L U C(G)^{*} ; \mathscr{L}_{g}^{0} \varphi=\varphi\right.$ for $\left.g \in G\right\}=\left\{\varphi \in L U C(G)^{*}\right.$; $\left.\mathscr{L}_{p_{n}}^{0} \varphi=\varphi, n=1,2, \cdots\right\}$ Denoting $L_{0}=L U C(G)$ we can apply Theorem II-1 to get that for any left invariant mean $\varphi$ of $L U C(G)^{*}$ there exists a sequence of finite means $\left\{\varphi_{n}\right\}$ such that $\lim _{n \rightarrow \infty} \varphi_{n}(f)=\varphi(f)$ for each $f \in L U C(G)$. We choose $\varphi$ as a two sided invariant mean on $m(G)$. (see [4] p. 529) This $\varphi$ will be fixed till the end of the proof. Then the restriction of this $\varphi$ (which we again denote by this same $\varphi$ ) to $L U C(G)$ will be at least a left invariant mean on $L U C(G)$. Therefore $\mathcal{D}(f)=\lim _{n \rightarrow \infty} \varphi_{n}(f)$ for each $f \in L U C(G)$ where $\varphi_{n}$ is a sequence of 
finite means of $L U C(G)^{* 6}$. Let $U$ be a neighborhood of $e$ such that none of its powers totally covers $G$. We may assume that $U$ is symmetric (since any neighborhood of $e$ included in $U$ also has this property). If $A \subset G$ we shall write $\varphi(A)$ instead of $\varphi\left(1_{A}\right)$ (we remember that $\varphi(f)$ is defined for any $f$ in $m(G)$ ). We shall show at first that $\varphi\left(U^{n}\right)>0$ for some integer $n>0$. This will immediately yield that $U^{2 n}$ totally covers $G$, which is the desired contradiction.

Define the following bounded uniformly continuous functions on the real line:

$$
f_{n}(x)= \begin{cases}1-2\left|x-\left(n-\frac{1}{2}\right)\right| & \text { if } n-1 \leqq x \leqq n \\ 0 & \text { otherwise }\end{cases}
$$

and

$$
g_{n+1}(x)= \begin{cases}1-2|x-n| & \text { if } n-\frac{1}{2} \leqq x \leqq n+\frac{1}{2} \\ 0 & \text { otherwise }\end{cases}
$$

Since the functions $f_{i}(x), f_{j}(x)$ (or $g_{i}(x), g_{j}(x)$ ) have disjoint carriers if $i \neq j$ the two functions $f(x)=\sum_{1}^{\infty} f_{n}(x)$ and $g(x)=\sum_{1}^{\infty} g_{n}(x)$ are well defined, their graph is plotted:

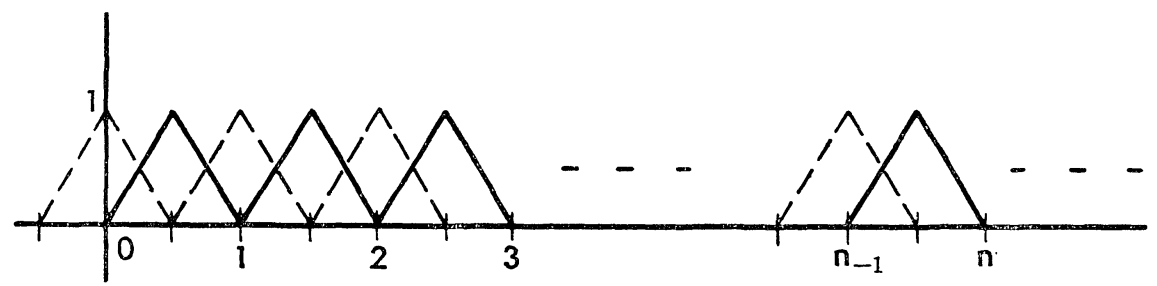

${ }^{6}$ This does not imply that $\varphi$ can be represented by a countably additive measure on the Baire field of $G$. Consider in fact the following example: Let $G$ be the additive group of rationals with the metric $\left|r_{2}-r_{1}\right|$ and let $a$ be an irrational number. Let $r_{n}$ be a sequence of rationals converging to $a$ and let $m_{n}$ be the point measure concentrated at $r_{n}$. Then $\lim _{n \rightarrow \infty} \int f d m_{n}=\lim _{n \rightarrow \infty} f\left(r_{n}\right)=A f$ exists for each uniformly continuous bounded $f$ (and equals $\widetilde{f}(a)$ where $\widetilde{f}$ is the uniformly continuous extension of $f$ to the whole real line). Assume now that $A f=\int f d m$ for some countably additive real valued measure $m$ on $B_{G}$ and consider the sequence of uniformly continuous functions defined for $x$ in $G$ by

$$
f_{n}(x)= \begin{cases}1-n|x-a| & \text { if }|x-a| \leqq \frac{1}{n} \\ 0 & \text { otherwise. }\end{cases}
$$

Then $f_{n}(x) \downarrow 0$ for each $x \in G$ and $\left|f_{n}(x)\right| \leqq 1$. Therefore $A f_{n}=\int f_{n} d m \rightarrow 0$ by Lebesgue's bounded convergence theorem. But $A f_{n}=\lim _{k \rightarrow \infty} f_{n}\left(r_{k}\right)=1$ for each $n$, which cannot be.

If though, $G$ would be a locally compact group then the above relation $\varphi(f)=$ $\lim _{n \rightarrow \infty} \varphi_{n}(f)$ would imply that $\varphi$ can be represented by a countably additive measure on $B_{G}$. (see Dieudonne: Sur le produit de composition Compositio Math. 1954 p. 28). In this particular case the proof of our theorem could be simplified. 
where $f(x)$ is represented by the solid line while $g(x)$ by the interrupted line. If $\left\{a_{n}\right\}$ is any bounded sequence of reals then it may easily be proved that both $\sum a_{n} f_{n}(x)$ and $\sum a_{n} g_{n}(x)$ are bounded uniformly continuous functions on the real line. Therefore if $F(g)$ is the left uniformly continuous real valued function on the group $G$ which satisfies.

$F^{-1}([O k]) \subset \bigcup_{j=1}^{k+2} U^{2(k+2)} p_{j}$ (see Lemma III-1) then surely $\sum a_{n} f_{n}(F(g)$ ) and $\sum a_{n} g_{n}(F(g))$ will both be bounded left uniformly continuous functions on $G$. But since $\sum_{1} f_{n}(x)+\sum_{1} g_{n}(x) \geqq d \geqq 0$ for some $d>0$, for each $x \geqq 0$ we have that $\sum f_{n}(F(g))+\sum g_{n}(F(g)) \geqq d \geqq 0$ for each $g$ of $G$. Therefore $\varphi\left[\sum f_{n}(F(g))+\sum g_{n}(F(g))\right]>0$ and so either

$$
\varphi\left[\sum f_{n}(F(g))\right]>0 \quad \text { or } \varphi\left[\sum g_{n}(F(g))\right]>0 \text {. }
$$

Assume therefore that $\varphi\left[\sum f_{n}(F(g))\right]>0$ (for the other case the proof is similar) and define the following linear positive functionals on the Banach space $m$ of all the bounded real sequences $\left\{a_{k}\right\}$ (with the sup norm):

$$
\varphi_{n}^{\prime}\left[a_{k}\right]=\varphi_{n}\left[\sum_{k=1}^{\infty} a_{k} f_{k}(F(g))\right]
$$

and

$$
\varphi^{\prime}\left\{a_{k}\right\}=\varphi\left[\sum_{k=1}^{\infty} a_{k} f_{k}(F(g))\right]
$$

where $\varphi_{n}$ is the sequence of finite means of $L U C(G)^{*}$ which satisfies $\lim _{n \rightarrow>} \varphi_{n}(f)=\varphi(f)$ for each $f$ in $L U C(G)$. But for any $f \in L U C(G)$.

$\varphi_{n}(f)=\sum_{i=1}^{j} \alpha_{i} f\left(g_{i}\right)$ where $\alpha_{i} \geqq 0 \sum \alpha_{i}=1$ (and $j,\left\{\alpha_{i}\right\}$ and $g_{i} \in G$ depend on $\varphi_{n}$.) Therefore as is easily seen

$$
\varphi_{n}^{\prime}\{1\}=\varphi_{n}\left(\sum_{k=1}^{\infty} f_{k}(F(g))\right)=\sum_{k=1}^{\infty} \varphi_{n} f_{k}(F(g))=\sum_{k=1}^{\infty} \varphi_{n}^{\prime}\left\{1_{k}\right\}
$$

where $\{1\} \in m$ is the sequence whose constant value is 1 and $\left\{1_{k}\right\} \in m$ is the sequence which is identically zero except at the place $k$ where it is 1 . This shows that $\varphi_{n}^{\prime} \in Q\left[l_{1}\right] \subset m^{*}$ where $l_{1}$ is the Banach space of all the absolute convergent real sequences $\left\{b_{i}\right\}$ with norm $\sum\left|b_{i}\right|$ and $Q: l_{1} \rightarrow l_{1}^{* *}=m^{*}$ is the natural maping from the Banach space $l_{1}$ into its second adjoint. (see Day [5] pp. 29-30). But $l_{1}$ is weakly sequentially complete ([5] p. 33 Cor. 3) and therefore $Q\left[l_{1}\right]$ is $w^{*}$ sequentially complete in $m^{*}$. (for notation see Day [5] p. 17). Therefore we have the following situation: If $n \rightarrow \infty$ then

$$
\varphi_{n}^{\prime}\left\{a_{k}\right\}=\varphi_{n}\left[\sum a_{k} f_{k}(F(g)] \rightarrow \varphi\left[\sum a_{k} f_{k}(F(g))\right]=\varphi^{\prime}\left\{a_{k}\right\} .\right.
$$

Thus $\varphi^{\prime} \in l_{1}$ which immediately implies that $\varphi^{\prime}\{1\}=\sum_{k=1}^{\infty} \varphi^{\prime}\left\{1_{k}\right\}$. But by definition $\varphi^{\prime}\left\{\mathbf{1}_{k}\right\}=\varphi\left[f_{k}(F(g))\right]$. Thus 


$$
0<\varphi\left[\sum f_{k}(F(g))\right]=\varphi^{\prime}\{1\}=\sum \varphi^{\prime}\left\{1_{k}\right\}=\sum \varphi f_{k}(F(g))
$$

and since $f_{k}(F(g)) \geqq 0$ for each $g$ in $G$ and $\varphi \geqq 0$ we have that for at least one $k>0, \varphi\left[f_{k}(F(g))\right]=c>0$. Now

$$
\left\{t ; f_{k}(t)>0\right\} \subset[k-1, k] \subset[0, k]
$$

and so

$$
\left\{g ; f_{k}(F(g))>0\right\} \subset\{g ; F(g) \in[0, k]\}=F^{-1}[0, k] \subset \bigcup_{j=1}^{k+2} U^{2(k+2)} p_{j} .
$$

But we can easily find (as in elementary integration theory) a function of the form $h(g)=\sum_{1}^{l} \alpha_{i} 1_{\Delta_{l}}(g) \in m(G)$ such that $\alpha_{i} \geqq 0 \alpha_{l}=0, A_{1}, \cdots, A_{l-1}$ form a partition of $\left\{g ; f_{k}(F(g))>0\right\}$ and $A_{l}=G-\left\{g ; f_{k}(F(g))>0\right\}$ and $0 \leqq f_{k}(F(g))-h(g)<c / 3$. If we remember now that $\varphi$ is defined on all of $m(G)$ (and we have used till now only its restriction to $L U C(G)$ ) we can write

$$
\begin{aligned}
c & =\varphi\left[f_{k}(F(g))\right] \\
& =\varphi\left[f_{k}(F(g))-h(g)\right]+\varphi[h(g)] \leqq c / 3+\varphi[h(g)] .
\end{aligned}
$$

Therefore $\varphi(h)>0$ which implies immediately that $\varphi\left(A_{i}\right)>0$ for some $1 \leqq i \leqq l-1$. Since $A_{i} \subset \bigcup_{j=1}^{k+2} U^{2(k+1)} p_{j}$ we get that $\varphi\left(U^{2(k+2)} p_{j}\right)>0$ for some $j$ and using the fact that $\varphi$ is also a right invariant mean we get that $\varphi\left(U^{2(k+2)}\right)>0\left(\right.$ Remember that $\varphi(A)=\varphi\left(1_{A}\right)=\varphi\left(1_{A}^{g}\right)=\varphi\left(1_{A^{-1}}\right)$ for any $g \in G$ and $A \subset G$ ).

Let now $V=U^{2(k+2)}$. We shall prove that $V^{2}=U^{4(k+2)}$ totally covers $G$, which will contradict the assumption that no power of $U$ totally covers $G . \quad U$ is symmetric and therefore so is $V$ and $\varphi(V)>0$. Assume that $V^{2}$ does not totally cover $G$. Then we chose an infinite sequence of elements $\left\{a_{n}\right\} \subset G$ this way: $a_{1}=e$. Since $G \neq V^{2} a_{1}$ let $a_{2} \notin V^{2} a_{1}$. Thus $V a_{2} \cap V a_{1}=\varnothing$ (since $V^{-1}=V$ ). If $a_{1}, \cdots, a_{n-1}$ have been chosen such that $V a_{i} \cap V a_{j}=\varnothing$ if $i \neq j$ and $1 \leqq i, j \leqq n-1$ then since $G \neq \bigcup_{1}^{n-1} V^{2} a_{i}$ there is some element $a_{n} \notin \bigcup_{1}^{n-1} V^{2} a_{i}$. Thus $a_{n} \notin V^{2} a_{i}$ for each $1 \leqq i \leqq n-1$ and so $V a_{n} \cap V a_{i}=\varnothing$ for $1 \leqq i \leqq n-1$. Therefore for any $n>0$

$$
1=\varphi(g) \geqq \varphi\left(V a_{1}\right)+\varphi\left(V a_{2}\right)+\cdots+\varphi\left(V a_{n}\right)=n \varphi(V) .
$$

This shows that $\varphi(V)=0$ which is a contradiction and so $V^{2}=U^{4(k+2)}$ totally covers $G$. This proves that $J_{u} l(G)$ is infinite dimensional. As an immediate consequence one gets that $J_{c} l(G)$ is infinite dimensional as follows: $G$ is amenable and so surely $\operatorname{dim} J_{c} l(G) \geqq 1$ and $\operatorname{dim} J_{x} l(G) \geqq 1$. Assume now that $\operatorname{dim} J_{c} l(G)=n, n<\infty$. We show that this implies that $\operatorname{dim} J_{u} l(G) \leqq n$, which cannot be.

If $\psi \in J_{u} l(G)$ then it has a left invariant extension $\psi^{\prime \prime} \in m(G)^{*}$ (see 
Remark II-2). The restriction $\psi^{\prime}$ of this $\psi^{\prime \prime}$, to $C(G)$ is left invariant and so any $\psi \in J_{u} l(G)$ has an extension $\psi^{\prime} \in J_{c} l(G)$. If $\operatorname{dim} J_{c} l(G)=n$, $n<\infty$, and $\left\{\psi_{1}, \cdots, \psi_{n+1}\right\} \subset J_{u} l(G)$ would be linearly independent then let $\left\{\psi_{1}^{\prime}, \cdots, \psi_{n+1}^{\prime}\right\} \subset J_{c} l(G)$ be respective extensions. Then $\sum_{1}^{n+1} \alpha_{1} \psi_{i}^{\prime}=0$ for some reals $\alpha_{i}$ would surely imply that $\sum_{1}^{n+1} \alpha_{i} \psi_{i}(f)=0$ for any $f$ in $L U C(G)$ and so $\alpha_{i}=0$ for $1 \leqq i \leqq n+1$. Therefore $\operatorname{dim} J_{u} l(G) \leqq n$ which cannot be.*

THeOREM 2. Let $G$ be a separable locally compact hausdorff topological group which is amenable as a discrete group. Let $J_{c} l(G) \subset C(G)^{*}$ be the space of left invariant elements of $C(G)^{*}, J_{u} l(G) \subset L U C(G)^{*}$ be the space of left invariant elements of $L U C(G)^{*}$. Then

(1) Either $\operatorname{dim} J_{c} l(G)=1$ or $\operatorname{dim} J_{c} l(G)=\infty$ and furthermore $\operatorname{dim} J_{c} l(G)=1$ if and only if $G$ is compact.

(2) Either $\operatorname{dim} J_{u} l(G)=1$ or $\operatorname{dim} J_{u} l(G)=\infty$ and furthermore $\operatorname{dim} J_{u} l(G)=1$ if and only if $G$ is compact.

REMARK 2. (a) The reader should remember that at least any abelian or solvable, or locally finite group is amenable as a discrete group. (see Day [4] pp. 516-518 for these and more examples)

(b) This theorem is not known even for the real line $R$. It asserts that $C(R)^{*}$ ahd $L U C(R)^{*}$ both have an infinite dimensional subspace of invariant elements.

Proof of theorem. $G$ is amenable and so the restriction of any left invariant mean to $C(G)$ or $L U C(G)$ is a left invariant mean of $C(G)$ or $L U C(G)$. Thus $\operatorname{dim} J_{c} l(G) \geqq 1$ and $\operatorname{dim} J_{u} l(G) \geqq 1$ in any case.

If $G$ is compact then $L U C(G)=C(G)$ as well known (see A. Weil [18]) and there is a unique left invariant mean on $C(G)$ (which is represented by the normalized Haar measure on $G$ ). Thus by the Remark II-2 we get that $\operatorname{dim} J_{u} l(G)=\operatorname{dim} J_{c} l(G)=1$.

Assume now that $\operatorname{dim} J_{u} l(G)=n, n<\infty$. Then $G$ is compact (since otherwise it would be noncompact locally compact and therefore would satisfy property $B$ and by the previous theorem would satisfy $\operatorname{dim} J_{u} l(G)=\infty$ ) Therefore $n=1$. Thus $\operatorname{dim} J_{u} l(G)$ can be either 1 or $\infty$ and $\operatorname{dim} J_{u} l(G)=1$ if and only if $G$ is compact. Using in the same way the previous theorem one immediately gets the remaining part of this theorem. Remembering that any nonzero subgroup of the

* In fact if $A$ is any left invariant subspace of $m(G)$ containing $L U C(G)$ and $J_{A} 1(G)$ the space of left invariant elements of $A^{*}$, then as above, $\operatorname{dim} J_{u} 1(G) \leqq$ $\operatorname{dim} J_{A} 1(G)$ which shows that Theorem III-1 holds true $C(G)$ is replaced by $A$. All the following theorems involving $C(G)$ could be shown to hold true when $C(G)$ is replaced by $A$. We could take as $A$, for instance, the space of all bounded Baire measurable functions on $G$. 
additive group of a hausdorff locally convex linear topological space has property (B) (see, Remark III-1 (b)) and using in the same way Theorem III-1 one immediately obtains.

THEOREM 3. Let $G$ be any separable subgroup of the additive group of a hausdorff locally convex linear topological space. Then

(1) Either $\operatorname{dim} J_{u} l(G)=1$ or $\operatorname{dim} J_{u} l(G)=\infty$ and furthermore $\operatorname{dim} J_{u} l(G)=1$ if and only if $G=\{0\}$.

(2) Either $\operatorname{dim} J_{c} l(G)=1$ or $\operatorname{dim} J_{c} l(G)=\infty$ and furthermore $\operatorname{dim} J_{c} l(G)=1$ if and only if $G=\{0\}$.

EXAMPLE 1. From the above theorems it follows that for separable locally compact groups (which are amenable as discrete groups) and for separable subgroups of a hausdorff locally convex linear topological space $\operatorname{dim} J_{c} l(G)=\operatorname{dim} J_{u} l(G)$ invariably holds. We give now on example of an abelin countable hausdorff topological group which satisfies $\operatorname{dim} J_{u} l(G)=1$ while $\operatorname{dim} J_{c} l(G)=\infty$. Let $G^{\prime}$ be a compact abelian separable metric group which is not finite and let $d(x, y)$ be an admissible invariant metric on $G^{\prime}$. Then $f \in L U C\left(G^{\prime}\right)$ if and only if $f$ is uniformly continuous on $G^{\prime}$ as a metric space with the metric $d$. Let $\left\{g_{1} g_{2}, \cdots\right\}$ be a countable dense subset of $G^{\prime}$ and let $G$ be the group generated by $\left\{g_{1} g_{2}, \cdots\right\}$. Then $G$ is a countable Hausdorff abelian topological group and therefore $G$ is $T_{1}$ and regular (even completely regular see [18] p. 13). Therefore $G$ is amenable as a discrete group and hence we can apply Corollary II-2 to get that $\operatorname{dim} J_{c} l(G)=\infty$.

Consider now $L U C(G)$. Any $f$ in $L U C(G)$ has a unique uniformly continuous extension $f^{\prime} \in C\left(G^{\prime}\right)$ such that $\sup _{g \in G}|f(g)|=\sup _{g \in G^{\prime}}\left|f^{\prime}(g)\right|$. But any $f^{\prime} \in C\left(G^{\prime}\right)$ is uniformly continuous on the (compact) metric space $\left(G^{\prime}, d\right)$ and therefore its restriction to $G$ is uniformly continuous on $(G, d)$. Thus $T: C\left(G^{\prime}\right) \rightarrow L U C(G)$ defined by $(T f)(g)=f(g)$ for $g$ in $G$ is a positive linear isometry onto $L U C(G)$. Therefore $T^{*}: L U C(G)^{*} \rightarrow$ $C\left(G^{\prime}\right)^{*}$ is an isometry. Since $\operatorname{dim} J_{c} l\left(G^{\prime}=1\right.$ it will be enough to show that $T^{*} \phi \in J_{c} l\left(G^{\prime}\right)$ for any $\varphi \in J_{u} l(G)$.

Let $l_{a}^{\prime}: C\left(G^{\prime}\right) \rightarrow C\left(G^{\prime}\right)$ be defined by $l_{a}^{\prime} f=f_{a}$ for $a \in S$ and $l_{a}^{0}: L U C(G) \rightarrow$ $L U C(G)$ be defined by $l_{a}^{0} f=f_{a}$ for $a \in S$. If $g, a \in G \subset G^{\prime}$ then

$$
T\left(l_{a}^{\prime} f\right)(g)=\left(l_{a}^{\prime} f\right)(g)=f(a g)=l_{a}^{0}(T f)(g) .
$$

Thus $T\left(l_{a}^{\prime} f\right)=l_{a}^{0}(T f)$ if $a \in G$. Let now $\varphi \in J_{u} l(G)$ and $a \in G$ then for $f \in C\left(G^{\prime}\right)\left(T^{*} \varphi\right)\left(l_{a}^{\prime} f\right)=\varphi\left(T l_{a}^{\prime} f\right)=\varphi\left(l_{a}^{0} T f\right)=\varphi(T f)=T^{*} \varphi(f)$.

If $a \notin G$ but $a \in G^{\prime}$ then there is a sequence $\left\{a_{n}\right\} \subset G$ such that $d\left(a_{n} a\right) \rightarrow 0$. Since $d$ is an invariant metric we have that $d\left(a_{n} g, a g\right)=$ $d\left(a_{n} a\right) \rightarrow 0$ for any $g$ in $G$. But any $f \in C\left(G^{\prime}\right)$ is uniformly continuous, which means that for $\varepsilon>0$ there is a $\delta>0$ such that if $d(x, y)<\delta$ then $|f(x)-f(y)|<\varepsilon$. If therefore $n_{0}$ is such that $n \geqq n_{0}$ implies 
$d\left(a_{n} a\right)<\delta$ then $\left|\left(l_{a_{n}}^{\prime}-l_{a}^{\prime}\right) f(g)\right|=\left|f\left(a_{n} g\right)-f(a g)\right|<\varepsilon$. This shows that $\left\|\left(l_{a_{n}}^{\prime}-l_{a}^{\prime}\right) f\right\| \rightarrow 0$ if $n \rightarrow \infty$. Thus

$$
\left(T^{*} \varphi\right)\left(l_{a}^{\prime} f\right)=\lim _{n \rightarrow \infty}\left(T^{*} \varphi\right)\left(l_{a_{n}}^{\prime} f\right)=\left(T^{*} \varphi\right) f
$$

since $a_{n} \in G$. Therefore $T^{*} \varphi \in J_{c} l\left(G^{\prime}\right)$. As one can easily see the condition that $G^{\prime}$ is metric is not essential and may easily be dropped. Also instead of $G^{\prime}$ being abelian we may require that $G^{\prime}$ is amenable as a discrete group and therefore we get:

COROLLARY 1. If $G_{1}$ is a compact hausdorff topological group which is amenable as a discrete group and $G \subset G_{1}$ is any countable (not finite) subgroup then $\operatorname{dim} J_{u} l(G)=1$ while $\operatorname{dim} J_{c} l(G)=\infty$.

We may remark that we take $G^{\prime}$ of the preceeding example to be the closure of $G$ in $G_{1}$ and we remember that $G^{\prime}$ as a subgroup of an amenable group is also amenable as a discrete group. (see Day [4] p. $516(\mathrm{D}))$.

Applications: The Banach Algebra $L U C(G)^{*}$. Let $G$ be a topological group and define in $L U C(G)^{*}$ (where $L U C(G) \subset C(G)$ are the left uniformly continuous functions with the sup. norm) the following multiplication: If $\varphi, \psi \in L U C(G)^{*}$ then for $f \in L U C(G)[\varphi \odot \psi](f)=$ $\varphi(y)$ where $y(h)=\psi\left(l_{h}^{0} f\right)$ for $h \in G$. (And $l_{a}^{0}: L U C(G) \rightarrow L U C(G)$ is defined by $l_{a}^{0} f=f_{a}$ for $\left.a \in G\right)$. The function $y$ belongs to $L U C(G)$. In fact $|y(h)| \leqq\|\psi\|\left\|l_{h}^{0} f\right\| \leqq\|\psi\|\|f\|$ and so $y$ is bounded, but moreover, $y(h)$ is left uniformly continuous. This is true since for any $\varepsilon>0$ there is a neighborhood of the identity $V$ such that $|f(v g)-f(g)|<\varepsilon$ for each $g$ in $G$ and $v$ in $V$. In other words $\left\|l_{v}^{0} f-f\right\|<\varepsilon$ for each $v$ in $V$. Thus

$$
\begin{aligned}
|y(v h)-y(h)| & =\left|\psi l_{v h}^{0} f-\psi l_{h}^{0} f\right|=\left|\psi\left(l_{v h}^{0} f-l_{h}^{0} f\right)\right| \\
& =\left|\psi l_{h}^{0}\left(l_{v}^{0} f-f\right)\right| \leqq\|\psi\|\left\|l_{v}^{0} f-f\right\|<\|\psi\| \varepsilon
\end{aligned}
$$

for each $h$ in $G$. Therefore this multiplication is at least well defined. But moreover, it renders $L U C(G)^{*}$ a Banach algebra as easily shown and known. In fact if $\varphi, \psi \in L U C(G)^{*}$ and $f \in L U C(G)$ then

$$
|(\varphi \odot \psi)(f)|=\left|\varphi_{h}\left(\psi l_{h}^{0} f\right)\right| \leqq\|\varphi\|\left\|\psi l_{h}^{0} f\right\|
$$

(where $\varphi_{h}$ means $\varphi$ with respect to the variable $h \in G$ and $\left\|\psi l_{h}^{0} f\right\|=$ $\left.\sup _{h \in G}\left|\psi l_{h}^{0} f\right|\right)$. But $\left|\psi l_{h}^{0} f\right| \leqq\|\psi\|\left\|l_{h}^{0} f\right\| \leqq\|f\|\|\psi\|$. The associative law is also easily proved. In fact if $\lambda, \mu, \nu \in L U C(G)^{*}$ and $f \in L U C(G)$ then $[\lambda \odot(\mu \odot \nu)](f)=\lambda_{a}\left[(\mu \odot \nu) l_{a}^{0} f\right]=\lambda_{a}\left[\mu_{b}\left[\nu l_{b}^{0}\left(l_{a}^{0} f\right)\right]\right]$. But

$$
[(\lambda \odot \mu) \odot \nu] f=(\lambda \odot \mu)_{b}\left[\nu l_{b}^{0} f\right]=(\lambda \odot \mu)(y)
$$


where $y(g)=\nu l_{g}^{0} f$ for each $g \in G$. But $\left(l_{a}^{0} y\right)(g)=y(a g)=\nu\left(l_{a g}^{0} f\right)=\nu\left(l_{g}^{0} l_{a}^{0} f\right)$. Therefore $(\lambda \odot \mu) y=\lambda_{a}\left[\mu l_{a}^{0} y\right]=\lambda_{a}\left[\mu_{b}\left(\nu l_{b}^{0} l_{a}^{0} f\right)\right]$ which implies that $\odot$ is associative. The distributive laws are also easily proved. The following should be noted here: In $C(G)^{*}$ we cannot define the same multiplication as above since if $\varphi, \psi \in C(G)^{*}, f \in C(G)$ and $G$ is not compact then $y(h)=\psi\left(f_{h}\right)$ is not generally a continuous function of $h$. In fact the following nice result has been established by Chivukula $R$. Rao, for groups $G$ with an invariant metric: If $f \in C(G)$ satisfies for each $\psi \in C(G)^{*}$ that $\psi f_{g}=y(g) \in C(G)$ then $f$ is uniformly continuous (see C. R. Rao [13] p. 17 thm 2). As an immediate consequence of our work combined with a result proved in Rudin [15], one gets the following results: (Denote by $R(G)$ the radical of the algebra $L U C(G)^{*}$.)

THEOREM 4. Let $G$ be a separable abelian locally compact hausdorff topological group. Then either $R(G)=\{0\}$ or $R(G)$ is infinite dimensional. Moreover; $R(G)=\{0\}$ (i.e. $L U C(G)^{*}$ is semisimple) if and only if $G$ is compact.

We need the following lemma whose proof is essentially known (see Civin-Yood [3], p. 849)

Lemma. Let $G$ be a topological group and $J_{u} l(G) \subset L U C(G)^{*}$ be the space of left invariant elements and let

$$
J_{1}=\left\{\varphi \in J_{u} l(G) ; \varphi\left(1_{G}\right)=0\right\} .
$$

Then $J_{1}$ is a two sided ideal and $J_{1}^{2}=\{0\}$.

Proof. If $\ell, \nu \in J_{1}$ and $f \in L U C(G)$ then $y(h)=\nu l_{h}^{0} f=\nu(f)$ for each $h \in G$ i.e., $y(h)=\nu(f) \cdot 1_{G}$ 。 Therefore $\mu \odot \nu(f)=\mu\left(\nu(f) \cdot 1_{G}\right)=$ $\nu(f) \mu\left(1_{G}\right)=0$. This shows that $J_{1}^{2}=\{0\}$. Let now $\varphi \in \operatorname{LUC}(G)^{*}$, $\nu \in J_{u} l(G)$ and $f \in L U C(G)$. Then $y(h)=\nu(f) 1_{G}(h)$. Thus $\varphi \odot \nu(f)=$ $\varphi(y)=\varphi\left(1_{G}\right) \cdot \nu(f)$. In other words

$$
\varphi \odot \nu=c \cdot \nu \text { where } c=\varphi\left(1_{G}\right) \text { is a constant . }
$$

If $\nu \in J_{1} \subset J_{u} l(G)$ then $(\varphi \odot \psi) 1_{G}=c \cdot \nu\left(1_{G}\right)=0$ and so $\varphi \odot \psi \in J_{1}$. Therefore $J_{1}$ is a left ideal. Moreover if $a \in G$ then

$$
\nu \odot \varphi\left(l_{a}^{0} f\right)=\nu_{h}\left(\varphi\left(l_{h}^{0} l_{a}^{0} f\right)\right)=\nu_{h}\left(\varphi l_{a h}^{0} f\right) .
$$

But if we define now $y(h)=\varphi\left(l_{h}^{0} f\right)$ then $\left(l_{a}^{0} y\right)(h)=y(a h)=\varphi\left(l_{a_{h}}^{0} f\right)$. Therefore $\nu_{h}\left(\varphi l_{a h}^{0} f\right)=\nu\left(l_{a}^{0} y\right)=\nu(y)=\nu\left(\varphi l_{h}^{0} f\right)=\nu \odot \varphi(f)$ which proves that $\nu \odot \varphi$ is left invariant. But since $l_{h}^{0} 1_{G}=1_{G}$ and $\varphi\left(l_{h}^{0} 1_{G}\right)=\varphi\left(1_{G}\right)=c$, we immediately get that $\nu \odot \varphi\left(1_{G}\right)=\nu\left(c 1_{G}\right)=c \cdot \nu\left(1_{G}\right)=0$. Therefore 
$\nu \odot \varphi \in J_{1}$ which finishes the proof of this lemma.

REMARK. The above lemma implies as well known that $J_{1} \subset R(G)$ for any topological group $G$.

Proof of Theorem 4. Denote by $M_{u} l(G)$ the set of left invariant means of $L U C(G)^{*}$ and let $\varphi_{0} \in M_{u} l(G)$ be fixed. Then obviously

$$
M_{u} l(G)-\varphi_{0}=\left\{\varphi-\varphi_{0}, \varphi \in M_{u} l(G)\right\} \subset J_{1} \subset R(G)
$$

since $\varphi\left(1_{G}\right)=1$ for each $\varphi \in M_{u} l(G)$. But as pointed out in the Remark (II-2) the linear manifold spanned by $M_{u} l(G)$ coincides with $J_{u} l(G)$. Assume now that $\operatorname{dim} R(G)=n$ where $0 \leqq n<\infty$, then $\operatorname{dim} J_{u} l(G)=$ $\operatorname{dim} M_{u} l(G)<\infty$. This implies by Theorem (III. 2 ) that $G$ is compact. But by Rudin [15] if $G$ is any compact abelian topological group then $C(G)^{*}$ with the above defined multiplication is semisimple. Since for compact $G, C(G)=L U C(G)$ we get that $R(G)=\{0\}$. Therefore either $R(G)=\{0\}$ or $\operatorname{dim} R(G)=\infty$. And $R(G)=\{0\}$ if and only if $G$ is compact.

THEOREM 5. If $G$ is separable subgroup of a locally convex linear topological space then either $R(G)=\{0\}$ or $\operatorname{dim} R(G)=\infty$. Moreover $R(G)=\{0\}$ if and only if $G=\{0\}$.

Proof. As in the previous theorem if $\operatorname{dim} R(G)=n$ where $0 \leqq n<\infty$ then $\operatorname{dim} J_{u} l(G)<\infty$ which implies by Theorem (III. 3) that $G=\{0\}$. But if $G=\{0\}$ then surely $R(G)=\{0\}$. Which finishes the proof of this theorem.

THEOREM 6. Let $G$ be a separable hausdorff topological group which is amenable as a discrete group. If $G$ has property (B) then $\operatorname{dim} R(G)=\infty$.

Proof. As above $M_{u} l(G)=\varphi_{0} \subset J_{1} \subset R(G)$. But by Theorem (III, 1) $\operatorname{dim} J_{u} l(G)=\infty$ and since $M_{u} l(G)$ spans $J_{u} l(G), \operatorname{dim} M_{u} l(G)=\infty$ which proves this theorem.

REMARK. (a) If $L U C(G)^{*}$ contains two distinct left invariant means $\varphi_{1}$ and $\varphi_{2}$ then the algebra $L U C(G)^{*}$ is not commutative since $\varphi_{1} \odot \varphi_{2}=\varphi_{2}$ and $\varphi_{2} \odot \varphi_{1}=\varphi$. Therefore if $G$ is even a commutative noncompact locally compact separable group, then $L U C(G)^{*}$ is not commutative.

(b) If $L_{c} U C(G)$ is the Banach space of bounded complex valued left uniformly continuous functions on $G$ and the algebra $L_{c} U C(G)^{*}$ is defined as above then Theorems III-4, III-5, III-6 hold true also 
for $L_{c} U C(G)^{*}$. Since any $\varphi \in J_{1}$ can be extended to $L_{c} U C(G)$ by defining for $f, g \in L U C(G) \varphi(f+i g)=\varphi(f)+i \varphi(g)$. If $J_{1}^{\prime} \subset L_{c} U C(G)^{*}$ is the set of all such extensions of elements of $J_{1} \subset L U C(G)^{*}$ then $J_{1}^{\prime} \subset R_{c}(G)$ where $R_{c}(G)$ denotes the radical of $L_{c} U C(G)^{*}$. From here one immediately gets that Theorem 4 holds also for the complex case.

\section{The invariant mean on semigroups containing compact groups and left ideals}

The main theorem of this chapter is Theorem IV-1. The following lemma is essentially known and we need it in the special form appearing here.

LeMMA 1. Let $S$ be a topological semigroup which contains a compact left-ideal group $A_{0}$. If $\left\{A_{\alpha} ; \alpha \in I\right\}$ is the set of all compact left-ideal groups of $S$ then $A=\bigcup_{\omega_{i}} A_{\alpha}$ is a right minimal ideal. Moreover if $e_{a}$ is the identity of the group $A_{a}$ then for any $a \in A$, $e_{\alpha} a=a$. Also for any $t \in S, t A_{\alpha}=A_{\alpha}$.

REMARK. $A_{\alpha}$ as groups and left ideals are minimal left ideals and therefore are disjoint.

Proof. Let $s \in S$. Then $A_{\alpha} s$ is a minimal left ideal since if $L \subset A_{\alpha} s$ is a left ideal and $a s \in L$ with $a \in A_{\alpha}$ then $A_{\alpha} s=\left(A_{\alpha} \alpha\right) s \subset L$ (since $A_{\alpha}$ is a group). Thus $A_{\alpha} s=L$ is a minimal left ideal. But $A_{\alpha} s a \subset A_{\alpha}$ for any $a \in A_{\alpha}$ and therefore $A_{\alpha} s a s \subset A_{\alpha} s$. Since $A_{\alpha} s$ is a minimal left ideal $\left(A_{\alpha} s\right) a s=A_{\alpha} s$. If $t \in S$ then $t A_{\alpha}=t\left(e_{\alpha} A_{\alpha}\right)=$ $\left(t e_{\alpha}\right) A_{\alpha}=A_{\alpha}$ since $t e_{\alpha} \in A_{\alpha}$, which is a group. In particular for $a \in A_{\alpha}$ $a s\left(A_{\alpha} s\right)=A_{\alpha} s$. In other words for any $b \in A_{\alpha} s, b\left(A_{\alpha} s\right)=A_{\alpha} s=\left(A_{\alpha} s\right) b$ holds which proves that the semigroup $A_{\alpha} s$ is in fact a group. Thus $A_{\alpha} s$ is a left ideal and group which as a continuous immage of $A_{\alpha}$ is also a compact subset of $S$. Therefore $A_{\alpha} s=A_{\beta} \subset A$ for some $\beta \in I$. Thus for any $s \in S, A s=\bigcup_{\alpha \in I} A_{\alpha} s \subset A$ which shows that $A$ is a right ideal.

Let now $R$ be any right ideal of $S$ and $r \in R$. Then $A_{\alpha}=r A_{\alpha} \subset R$ for each $\alpha \in I$. This shows that $A \subset R$ (i.e. that $A$ is included in each right ideal of $S$ ) and in particular that $A$ is a minimal right ideal. Now if $e_{\alpha}, e_{\beta}$ are the identities of $A_{\alpha}, A_{\beta}$ respectively then $e_{\alpha} \cdot e_{\beta} \in A_{\beta}$ and

$$
\left(e_{\alpha} e_{\beta}\right)\left(e_{\alpha} e_{\beta}\right)=e_{\alpha}\left(e_{\beta}\left(e_{\alpha} e_{\beta}\right)\right)=e_{\alpha}\left(e_{\alpha} e_{\beta}\right)=e_{\alpha} e_{\beta} .
$$

Thus $e_{\alpha} e_{\beta}$ is an idempotent of the group $A_{\beta}$ and therefore $e_{\alpha} e_{\beta}=e_{\beta}$ for any $\alpha, \beta \in I$. If now $\alpha \in A$ then $\alpha \in A_{\beta}$ for some $\beta \in I$ and therefore $e_{\alpha} \alpha=e_{\alpha}\left(e_{\beta} \alpha\right)=e_{\beta} \alpha=\alpha$. 
REMARK. In semigroup terminology this shows that $A$ is the Suschevitch kernel of the semigroup $S$.

If $\varphi \in C(S)^{*}$ then $\varphi \geqq 0$ (is positive) if $\varphi(f) \geqq 0$ for each $f \geqq 0$, $f \in C(S)$. An operator $T: C(S)^{*} \rightarrow C\left(S^{\prime}\right)^{*}$ is called positive if $T \varphi \geqq 0$ whenever $\varphi \geqq 0$.

Lemma 2. Let $S$ be the semigroup of Lemma $(\mathrm{IV}, 1)$ and $\pi: C(S) \rightarrow$ $C(A)$ be defined by $(\pi f)(a)=f(a)$ for $a$ in $A$. Then $\pi^{*}: C(A)^{*} \rightarrow C(S)^{*}$ is a linear positive isometry such that $\pi^{*}\left[J_{c} l(A)\right]=J_{c} l(S)$. Moreover $\pi^{*^{-1}}: J_{c} l(S) \rightarrow J_{c} l(A)$ is also positive.

Proof. $\pi$ maps $C(S)$ onto $C(A)$ since if $h \in C(A)$ then define $\tilde{h} \in C(S)$ by

(IV. 1)

$$
\widetilde{h}(s)=h\left(e_{\alpha} s\right),
$$

where $e_{\alpha}$ is the identity of the group $A_{\alpha}$ for some fixed $\alpha \in I$. If $s \in A$ then by the proceeding lemma $e_{\alpha} s=s$ and so $\tilde{h}(s)=h(s)$. Also $s \rightarrow e_{\alpha} s$ is a continuous map from $S$ to $A$ (with the relative topology) since if $0^{\prime}$ is open in $A$ then $0^{\prime}=0 \cap A$ with 0 open in $S$ and (since $A$ is a right ideal) $\left\{s ; e_{\alpha} s \in 0^{\prime}\right\}=\left\{s ; e_{\alpha} s \in 0\right\}$ which is open by the continuity of the left multiplication. Since $h \in C(A)$ we get that $h\left(e_{x} s\right)=\widetilde{h}(s) \in C(S)$. We also remark that if $\|h\| \leqq 1$ then $\|\widetilde{h}\| \leqq 1$ and so $\pi$ maps the unit ball of $C(S)$ onto the unit ball of $C(A)$. Also if $f \geqq 0$ then $\pi f \geqq 0$ and $\pi\left(1_{S}\right)=1_{A}$. Therefore if $\varphi \in C(A)^{*}$ then

$$
\begin{aligned}
\left\|\pi^{*} \varphi\right\| & =\sup _{\|f\| \leqq 1}\left|\left(\pi^{*} \varphi\right) f\right|=\sup _{\| f(S)}|\varphi(\pi f)| \\
& =\sup _{\|h\| \leqq 1}|\varphi(h)|=\|\varphi\|(A) \\
& \mid \varphi .
\end{aligned}
$$

Therefore $\pi^{*}: C(A)^{*} \rightarrow C(S)^{*}$ is a positive linear isometry into $C(S)^{*}$. We shall show that it maps $J_{c} l(A)$ onto $J_{c} l(S)$. If $s \in S, a \in A$ then let $l_{a}^{\prime}: C(A) \rightarrow C(A)$ and $l_{s}: C(S) \rightarrow C(S)$ be defined by: $l_{a}^{\prime} h=h_{a}$ and $l_{s} f=f_{s}$ for and $a \in A$ and $s$ in $S$. Let $\mathscr{L}_{s}=l_{s}^{*}$. Then $\left(\pi l_{a} f\right)(b)=$ $\left(l_{a} f\right)(b)=f(a b)=(\pi f)(a b)=\left(l_{a}^{\prime}(\pi f)\right)(b)$ for each $a, b \in A$. Thus $\pi l_{a} f=$ $l_{a}^{\prime} \pi f$ for each $f \in C(S)$ and so for any $a \in A$ and $\varphi \in J_{c} l(A)$ and $f \in C(S)$ :

$$
\left(\pi^{*} \varphi\right)\left(l_{a} f\right)=\varphi\left(\pi l_{a} f\right)=\varphi\left(l_{a}^{\prime} \pi f\right)=\varphi(\pi f)=\left(\pi^{*} \varphi\right)(f) .
$$

Thus $\mathscr{L}_{a}\left(\pi^{*} \phi\right)=\pi^{*} \phi$ for each $a \in A$. If now $s \in S$ and $a \in A$ then

$$
\mathscr{L}_{s}\left(\pi^{*} \varphi\right)=\mathscr{L}_{s}\left(\mathscr{L}_{a}\left(\pi^{*} \varphi\right)\right)=\mathscr{L}_{s a}\left(\pi^{*} \varphi\right)=\pi^{*} \varphi
$$

since $s a \in A$. Thus $\left.\pi^{*}: J_{c} l(A)\right) \rightarrow J_{c} l(S)$ is a linear positive isometry into. We prove now that $\pi^{*}$ maps $J_{c} l(A)$ onto $J_{c} l(S)$.

Let $\varphi \in J_{c} l(S)$ and let $f \in C(S)$ satisfy $f(a)=0$ for each $a \in A$. Then for $a \in A$ we have $\left(l_{a} f\right)(s)=f(a s)=0$, since $A$ is a right ideal. 
Thus $\varphi(f)=\varphi\left(l_{a} f\right)=\varphi(0)=0$. Therefore if $f_{1}, f_{2} \in C(S)$ satisfy $f_{1}(a)=$ $f_{2}(\alpha)$ for each $\alpha \in A$ then $f_{1}(\alpha)-f_{2}(\alpha)=0$ for $\alpha \in A$ and so $\varphi\left(f_{1}\right)=\varphi\left(f_{2}\right)$. In other words if $h \in C(A)$ and $\widetilde{h} \in C(S)$ is any extension of $h$ to all of $S$ then $\varphi(h)$ does not depend on the particular extension $\tilde{h} \in C(S)$ of $h \in C(A)$. Therefore $\varphi^{\prime} \in C(A)^{*}$ defined for $h \in C(S)$ by

$$
\varphi^{\prime}(h)=\varphi(\tilde{h})
$$

where $\tilde{h} \in C(S)$ is any extension of $h$ to all of $S$, is at least well defined. Moreover if $\varphi \geqq 0$ and $h \geqq 0$ then the extension $\tilde{h} \in C(S)$ defined above (IV. 1) satisfies $\widetilde{h}(s)=h\left(e_{\alpha} s\right) \geqq 0$ and so $\varphi^{\prime}(h)=\varphi(h) \geqq 0$. This shows that if $\varphi \geqq 0$ then $\varphi^{\prime} \geqq 0$. It is easily checked that $\varphi^{\prime}$ is linear. Also if $\|h\| \leqq 1$ then the extension defined by IV-1 satisfies $\|\tilde{h}\| \leqq 1$ and thus

$$
\left|\varphi^{\prime}(h)\right|=|\varphi(\tilde{h})| \leqq\|\varphi\|\|\tilde{h}\| \leqq\|\varphi\| .
$$

This shows that $\varphi^{\prime} \in C(A)^{*}$.

We show now that $\varphi^{\prime} \in J_{c} l(A)$. Let $a \in A$ be fixed. Then

$$
\varphi^{\prime}\left(l_{a}^{\prime} h\right)=\varphi\left(\widetilde{l_{a}^{\prime} h}\right)
$$

where $\widetilde{l_{a} h}$ is any extension, in $C(S)$, of $l_{a}^{\prime} h \in C(A)$. But $a \in A_{\alpha_{0}}$ for some $\alpha_{0} \in I$ and the function defined by

$$
\left(\widetilde{l_{a}^{\prime} h}\right)(s)=\left(l_{a}^{\prime} h\right)\left(e_{\alpha_{0}} s\right)
$$

is a bounded continuous extension of $l_{a}^{\prime} h \in C(A)$. (where $e_{\alpha_{0}}$ is the identity of $A_{\alpha_{0}}$ ). And for each $s \in S$ :

$$
\widetilde{l_{a}^{\prime} h}(s)=\left(l_{a}^{\prime} h\right)\left(e_{\alpha_{0}} s\right)=h\left(\alpha e_{\alpha_{0}} s\right)=h(\alpha s) .
$$

But if $\tilde{h} \in C(S)$ is any extension of $h$ to all of $S$ then, since $A$ is a right ideal, we get

$$
h(\alpha s)=\tilde{h}(\alpha s)=\left(l_{a} \tilde{h}\right)(s) .
$$

Therefore

$$
\widetilde{l_{a}^{\prime} h}(s)=\left(l_{a} \tilde{h}\right)(s)
$$

where $\widetilde{l_{a}^{\prime} h}$ is the extension defined by (IV. 2) while $\tilde{h} \in C(S)$ is any extension of $h$. Therefore

$$
\varphi^{\prime}\left(l_{a}^{\prime} h\right)=\varphi\left(\widetilde{l_{a}^{\prime} h}\right)=\varphi\left(l_{a} \widetilde{h}\right)=\varphi(\widetilde{h})=\varphi^{\prime}(h) .
$$

This shows that $\varphi^{\prime} \in J_{c} l(A)$. Moreover $\pi^{*} \varphi^{\prime}=\varphi$. In fact if $f \in C(S)$ then $\left(\pi^{*} \varphi^{\prime}\right)(f)=\varphi^{\prime}(\pi f)=\varphi(f)$ since $f$ is obviously an extension of $\pi f \in C(A)$. Therefore $\pi^{*}: J_{c} l(A) \rightarrow J_{c} l(S)$ is a positive linear isometry onto and positive elements in $J l(S)$ have positive preimages in $J_{c} l(A)$ 
or in other words $\pi^{*^{-1}}: J_{c} l(S) \rightarrow J_{c} l(A)$ is a linear positive isometry onto.

REMARK. We notice that we do not assume any separation axioms about the topological space $A$. We shall show in what follows that in fact we can assume about $A$ that it is even a hausdorff space (and even that $C(A)$ separates points).

In fact define in $A$ the following equivalence relation: If $a, b \in A$ then $a \sim b$ if and only if $x(a)=x(b)$ for each $x \in C(A)$. Obviously this is an equivalence relation but moreover $\sim$ is even a congruence, i.e., if $a \sim b$ then $c a \sim c b$ and $a c \sim b c$ for each $c \in A$. This is true since for any $x \in C(A)$

$$
x(c a)=x_{c}(a)=x_{c}(b)=x(c b)
$$

and

$$
x(a c)=x^{c}(a)=x^{c}(b)=x(b c) .
$$

Let $A^{\prime}$ be the collection of all equivalence classes of $A$ and for each $a \in A$ let $\alpha^{\prime}$ be the equivalence class containing $\alpha$. Define in $A^{\prime}$ the multiplication $a^{\prime} \cdot b^{\prime}=(a b)^{\prime}$. Since $\sim$ is a congurence this multiplication is well defined and renders $A^{\prime}$ a semigroup. (see Lyapin [20] p. 361-362). Thus $\psi: A \rightarrow A^{\prime}$ defined by $\psi(a)=a^{\prime}$ is a homomorphism of $A$ onto $A^{\prime}$. Define now in $A^{\prime}$ the quotient topology this way: $U^{\prime} \subset A^{\prime}$ is open if and only if $\psi^{-1}\left(U^{\prime}\right) \subset A$ is open. Thus $\psi: A \rightarrow A^{\prime}$ is a continuous homomorphism and so $A_{a}^{\prime}=\psi\left(A_{a}\right)$ are compact. Moreover if $a \in A_{a}$ then $A_{a}^{\prime}=\psi\left(A_{\alpha}\right)=\psi\left(a A_{\alpha}\right)=\psi(a) \psi\left(A_{\alpha}\right)=a^{\prime} A_{a}^{\prime}$ and in the same way $A_{\alpha}^{\prime} a^{\prime}=A_{\alpha}^{\prime}$ which shows that $A_{\alpha}^{\prime}$ is a group. Also if $b \in A$ then $b^{\prime} A_{\alpha}^{\prime}=\psi(b) \psi\left(A_{\alpha}\right)=\psi\left(b A_{\alpha}\right)=\psi\left(A_{\alpha}\right)=A_{\alpha}^{\prime}$ which shows that $A_{\alpha}^{\prime}$ is a left ideal.

But moreover, $A^{\prime}$ with the above defined quotient topology has separately continuous multiplication. In fact if $U^{\prime}$ is an open set in $A^{\prime}$ and $a_{0} \in A$ then we have to show that $0^{\prime}=\left\{c^{\prime} ; a_{0}^{\prime} c^{\prime} \in U^{\prime}\right\}$ is open in $A^{\prime}$ or that

$$
0=\psi^{-1}\left(0^{\prime}\right)=\left\{c ;\left(a_{0} c\right)^{\prime} \in U^{\prime}\right\}=\left\{c ; a_{0} c \in \psi^{-1}\left(U^{\prime}\right)\right\}
$$

is open in $A$. But since $\psi$ is continuous $\psi^{-1}(U)$ is open in $A$ and since left multiplication by $a_{0}$ is continuous, we get that $\left\{c ; a_{0} c \in \psi^{-1}\left(U^{\prime}\right)\right\}$ is open in $A$. In the same way one shows that right multiplication in $A^{\prime}$ is continuous. Define now the map $\widetilde{\psi}: C\left(A^{\prime}\right) \rightarrow C(A)$ by $\left(\widetilde{\psi} x^{\prime}\right)(a)=$ $x^{\prime}(\psi a)=x^{\prime}\left(a^{\prime}\right)$ for each $a \in A$. Since $\psi(A)=A^{\prime}, \tilde{\psi}$ is a linear positive isometry (i.e., if $x^{\prime} \geqq 0$ then $\widetilde{\psi}\left(x^{\prime}\right) \geqq 0$ ) into $C(A)$. But we notice now that each $x \in C(A)$ gives raise to an $x^{\prime} \in C\left(A^{\prime}\right)$ by defining: $x^{\prime}\left(\alpha^{\prime}\right)=x(\alpha)$ where $a$ is any representative of the equivalence class $a^{\prime} \in A^{\prime}$. Since $x$ 
is constant on equivalence classes, $x^{\prime}$ is well defined and $x^{\prime} \in C\left(A^{\prime}\right)$, since if $V$ is an open set of reals then

$$
\psi^{-1}\left\{a^{\prime} ; x^{\prime}\left(a^{\prime}\right) \in V\right\}=\left\{a ; x(a)=x^{\prime}\left(a^{\prime}\right) \in V\right\}=x^{-1}(V)
$$

which is open in $A$ since $x \in C(A)$. Also $\left(\widetilde{\psi} x^{\prime}\right)(a)=x^{\prime}(\psi(a))=x^{\prime}\left(a^{\prime}\right)=$ $x(a)$. This shows that $\widetilde{\psi}: C\left(A^{\prime}\right) \rightarrow C(A)$ is onto. It is immediate now that $A^{\prime}$ is hausdorff. In fact if $a^{\prime}, b^{\prime} \in A^{\prime}$ are such that $a^{\prime} \neq b^{\prime}$ then there is an $x \in C(A)$ such that $x(a) \neq x(b)$ i.e. $x^{\prime}\left(a^{\prime}\right) \neq x^{\prime}\left(b^{\prime}\right)$ so that $C\left(A^{\prime}\right)$ even separates points.

Lemma 3. $\widetilde{\psi}^{*}: C(A)^{*} \rightarrow C\left(A^{\prime}\right)^{*}$ is a linear positive isometry such ${ }_{\mathrm{E}}^{\mathrm{g}}$ that $\widetilde{\psi}^{*}\left[J_{c} l(A)\right]=J_{c} l\left(A^{\prime}\right) . \quad \widetilde{\psi}^{*-1}: J_{c} l\left(A^{\prime}\right) \rightarrow J_{c} l(A)$ is also positive.

Proof. Since $\widetilde{\psi}: C(A) \rightarrow C\left(A^{\prime}\right)$ is a positive isometry onto we immediately get that $\psi^{*}: C(A)^{*} \rightarrow C\left(A^{\prime}\right)^{*}$ is a linear positive isometry. Let now $l_{a^{\prime}}^{\prime}: C\left(A^{\prime}\right) \rightarrow C\left(A^{\prime}\right)$ be given by $\left(l_{a^{\prime}}^{\prime} x^{\prime}\right)\left(c^{\prime}\right)=x^{\prime}\left(a^{\prime} c^{\prime}\right)$ for each $c^{\prime} \in A^{\prime}$ and $l_{a}: C(A) \rightarrow C(A)$ by $l_{a} x=x_{a}$, As known and easily checked $l_{a}\left(\widetilde{\psi} x^{\prime}\right)=\widetilde{\psi}\left(l_{a}^{\prime}, x^{\prime}\right)$, which shows that if $\varphi \in J_{c} l(A)$ then:

$$
\left(\widetilde{\psi}^{*} \varphi\right)\left(l_{a^{\prime}}^{\prime} x^{\prime}\right)=\varphi \widetilde{\psi}\left(l_{a^{\prime}}^{\prime} x^{\prime}\right)=\varphi\left(l_{a} \widetilde{\psi} x^{\prime}\right)=\varphi\left(\widetilde{\psi} x^{\prime}\right)=\left(\widetilde{\psi}^{*} \varphi\right)\left(x^{\prime}\right) .
$$

Therefore $\left.\widetilde{\psi}^{*}\left[J_{c} l(A)\right] \subset J_{c} l\left(A^{\prime}\right)\right]$.

If now $\varphi^{\prime} \in J_{c} l\left(A^{\prime}\right)$ then let $\varphi \in C(A)^{*}$ be defined, for $x \in C(A)$, by $\varphi(x)=\phi^{\prime}\left(x^{\prime}\right)$ where $x^{\prime} \in C\left(A^{\prime}\right)$ is given by $x^{\prime}\left(a^{\prime}\right)=x(a)$ for each $a \in A$. Then $\left(l_{a} x\right)^{\prime}\left(b^{\prime}\right)=\left(l_{a} x\right)(b)=x(a b)=x^{\prime}\left((a b)^{\prime}\right)=x^{\prime}\left(a^{\prime} b^{\prime}\right)=\left(l_{a^{\prime}}^{\prime} x^{\prime}\right)\left(b^{\prime}\right)$. Thus

$$
\varphi\left(l_{a} x\right)=\varphi^{\prime}\left(\left(l_{a} x\right)^{\prime}\right)=\varphi^{\prime}\left(l_{a^{\prime}}^{\prime} x^{\prime}\right)=\varphi^{\prime}\left(x^{\prime}\right)=\varphi(x) .
$$

Therefore $\varphi \in J_{c} l(A)$. But $\left(\widetilde{\psi} x^{\prime}\right)(a)=x^{\prime}\left(a^{\prime}\right)=x(a)$ and thus

$$
\left(\widetilde{\psi}^{*} \varphi\right)\left(x^{\prime}\right)=\varphi\left(\widetilde{\psi} x^{\prime}\right)=\varphi(x)=\varphi^{\prime}\left(x^{\prime}\right) .
$$

This shows that $\widetilde{\psi}^{*} \varphi=\varphi^{\prime}$ i.e., that $\widetilde{\psi}^{*}\left[J_{c} l(A)\right]=J_{c} l\left(A^{\prime}\right)$. We also notice that if $\varphi^{\prime} \geqq 0$ then $\varphi \geqq 0$ and so positive elements in $J l\left(A^{\prime}\right)$ have positive preimages and so $\psi^{*-1}$ is also positive.

Remarks. We notice that $A_{\alpha}^{\prime}$ is a group which is a compact hausdorff topological space with separately continuous multiplication and therefore by Ellis theorem (see Ellis [21] or Glicksberg Deleeuw [19] p. 64-65 and p. 94-96) each $A_{\alpha}^{\prime}$ is a compact Hausdorff topological group. (i.e. the mapping $(a, b) \rightarrow a b^{-1}$ from $A_{a}^{\prime} \times A_{a}^{\prime}$ into $A_{\alpha}^{\prime}$ is continuous).

THEOREM 1. Let $S$ be a topological semigroup (only with separately continuous multiplication) and let $S$ contain exactly $n$ compact left-ideal groups $A_{1}, \cdots, A_{n}$. Then $\operatorname{dim} J_{c} l(S)=n$ and $J_{c} l(S)$ is 
spanned by the left invariant means.

Proof. If $A=\bigcup_{i=1}^{n} A_{i}$ then $\pi^{*}: J_{c} l(A) \rightarrow J_{c} l(S)$ is a positive isometry onto (and so maps left invariant means into left invariant means). If $A^{\prime}$ is the semigroup of Lemma (IV. 3) then $\widetilde{\psi}^{*-1}: J_{c} l\left(A^{\prime}\right) \rightarrow J_{c} l(A)$ is a linear positive isometry onto and so it is enough to show that $\operatorname{dim} J_{c} l\left(A^{\prime}\right)=n$ and that $J_{c} l\left(A^{\prime}\right)$ is spanned by the set of left invariant means. We recall now that $A^{\prime}=\bigcup_{i=1}^{n} A_{i}^{\prime}$ is a Hausdorff topological space and that $A_{i}^{\prime}$ are compact topological groups and left ideals and therefore disjoint. Thus $A^{\prime}$ is a compact hausdorff semigroup and multiplication is (at least) separately continuous. In what follows we shall drop the prime and write $A, A_{i}$ instead of $A^{\prime}, A_{i}^{\prime} \cdot A, A_{i}$ are compact hausdorff. But $A_{i}$ as the complement of the compact set $\bigcup_{j \neq i} A_{j}$, is also open. Therefore $1_{A_{i}} \in C(A)$. Hence if $f \in C(A)$ then $f(a)=$ $\sum f(a) 1_{A_{i}}(a)$ for each $a \in A$ and $f \cdot 1_{A_{i}} \in C(A)$. Moreover if $h \in C\left(A_{i}\right)$ then $\widetilde{h}$ defined by $\widetilde{h}(a)=h\left(a e_{i}\right)$ for each $a \in A$ is an extension of $h$ to all of $A$ and $\widetilde{h} \in C(A)$. Furthermore, if $h \geqq 0$ then $\widetilde{h} \geqq 0$ and if $\|h\| \leqq 1$ then $\|\tilde{h}\| \leqq 1$. Let $\pi_{i}: C(A) \rightarrow C\left(A_{i}\right)$ be defined by $\left(\pi_{i} f\right)(a)=$ $f(a)$ for $a \in A_{i}$. If $a \in A_{i}$ then let $l_{a}^{i}$ : $C\left(A_{i}\right) \rightarrow C\left(A_{i}\right)$ be defined by $l_{a}^{i} h=h_{a}$ for $a \in A_{i}$. Also, $l_{a}: C(A) \rightarrow C(A)$ is defined by $l_{a} f=f_{a}$ for any a in $A$. Let $l_{a}^{*}=\mathscr{L}_{a}$. Then as easily checked: $\pi_{i} l_{a} f=l_{a}^{i} \pi_{i} f$ for each $f \in C(A)$ and $a$ in $A_{i}$.

Let now $\varphi_{i}^{\prime} \in C\left(A_{i}\right)^{*}$ be the linear positive functional of norm one represented by the normalized Haar measure on the compact hausdorff topological group $A_{i}$. Define $\varphi_{i} \in C(A)^{*}$ by

$$
\varphi_{i}(f)=\varphi_{i}^{\prime}\left(\pi_{i} f\right) \quad \text { for each } f \in C(A) .
$$

Then we get immediately that $\varphi_{i} \geqq 0, \varphi_{i}\left(1_{A}\right)=1$ and that $\varphi_{i}\left(1_{A_{i}}\right)=1$ while $\varphi_{i}\left(1_{A_{k}}\right)=0$ if $i \neq k$. Thus for any $a \in A_{i}$ :

$$
\varphi_{i}\left(l_{a} f\right)=\varphi_{i}^{\prime}\left(\pi_{i} l_{a} f\right)=\varphi_{i}^{\prime}\left(l_{a}^{i} \pi_{i} f\right)=\varphi_{i}^{\prime}\left(\pi_{i} f\right)=\varphi_{i}(f) .
$$

Therefore $\mathscr{L}_{a} \varphi_{i}=\varphi_{i}$ for each $a \in A_{i}$. If now $c \in A$ and $a \in A_{i}$ then $\mathscr{L}_{c} \varphi_{i}=\mathscr{L}_{c} \mathscr{L}_{a} \varphi_{i}=\mathscr{L}_{c a} \varphi_{i}=\varphi_{i}$ since $c a \in A_{i}$. Therefore $\varphi_{i}$ is a left invariant mean in $C(A)^{*}$. Also $\varphi_{1}, \cdots, \varphi_{n}$ are linearly independent (since if $\sum \alpha_{i} \varphi_{i}=0$ then $\alpha_{k}=\left(\sum \alpha_{j} \varphi_{j}\right)\left(1_{A_{k}}\right)=0$ ). It remains to show that $\varphi_{1}, \cdots, \varphi_{n}$ span $J_{c} l(A)$.

If $h \in C\left(A_{i}\right)$ and if $\widetilde{h} \in C(A)$ is any extension of $h$ (for instance $h(c)=h\left(c e_{i}\right)$ for each $\left.c \in A\right)$ then let $P_{i}(h)=\widetilde{h} \cdot 1_{A_{i}} \in C(A)$. In other words $P_{i} h \in C(A)$ equals $h$ on $A_{i}$ and 0 outside $A_{i}$. Thus $P_{i}: C\left(A_{i}\right) \rightarrow$ $C(A)$ and as easily checked:

$$
\pi_{i} P_{i} h=h
$$

for $h \in C\left(A_{i}\right)$

and 
(IV. 5)

$$
P_{i} \pi_{i} f=f 1_{A_{i}} \quad \text { for } f \in C(A) .
$$

If $a \in A_{i}$ and $h \in C\left(A_{i}\right)$ then

$$
P_{i}\left(l_{a}^{i} h\right)(b)=\left\{\begin{array}{ll}
0 & \text { if } b \notin A_{i} \\
\left(l_{a}^{i} h\right)(b)=h(a b) & \text { if } b \in A_{i}
\end{array} .\right.
$$

Moreover,

$$
l_{a}\left(P_{\imath} h\right)(b)=\left(P_{i} h\right)(a b)=\left\{\begin{array}{ll}
0 & \text { if } a b \notin A_{i} \\
h(a b) & \text { if } a b \in A_{i}
\end{array} .\right.
$$

But $a b \in A_{i}$ if and only if $b \in A_{i}$ (if $b \notin A_{i}$ then $b \in A_{j}$ for $j \neq i$ and so $a b \in A_{j}$ ) and $a b \notin A_{i}$ if and only if $b \notin A_{i}$. This shows that

$$
P_{i}\left(l_{a}^{i} h\right)=l_{a}\left(P_{i} h\right) \quad \text { for each } h \in C\left(A_{i}\right) \text { and } a \in A_{i} .
$$

Let $\varphi \in J_{c} l(A) \subset C(A)^{*}$ and define $\psi_{i} \in C\left(A_{\imath}\right)^{*}$ by

$$
\psi_{i}(h)=\varphi\left(P_{i} h\right) .
$$

If $a \in A_{i}$ then by IV-6, IV-7: $\psi_{i}\left(l_{a}^{i} h\right)=\varphi\left(P_{i} l_{a}^{i} h\right)=\varphi\left(l_{a} P_{i} h\right)=\varphi\left(P_{i} h\right)=$ $\psi_{i}(h)$ which shows that $\psi_{i}$ is a left invariant functional in $C\left(A_{i}\right)$. Therefore, (by the uniqueness of the Haar measure) we get that $\psi_{i}=$ $\alpha_{i} \varphi_{i}^{\prime}$ for some real number $\alpha_{i}$. Therefore if $f \in C(A)$ then using IV-5, IV-7 and IV-3 one gets:

$$
\begin{aligned}
\varphi(f) & =\sum \varphi\left(f 1_{A i}\right)=\sum \varphi\left(P_{i} \pi_{\imath} f\right) \\
& =\sum \psi_{i}\left(\pi_{i} f\right)=\sum \alpha_{i} \varphi_{i}^{\prime}\left(\pi_{i} f\right)=\sum \alpha_{i} \varphi_{i}(f) .
\end{aligned}
$$

Thus $\varphi=\sum \alpha_{i} \varphi_{i}$ which finishes the proof. As a special case one gets the following theorem of I. S. Luthar (see [12] p. 403).

THEOREM. If $S$ is an abelian topological semigroup which contains a compact ideal then $\operatorname{dim} J_{c} l(S)=1$.

Proof. As in Luthar's proof if $I$ is a compact ideal of $S$ and $I_{1}, \cdots, I_{n}$ are closed ideals of $S$ contained in $I$ then $I_{1} \cdots I_{n} \subset \bigcap_{j=1}^{n} I_{j} \neq \varnothing$. Therefore the family $F$ of all closed ideals of $S$ contained in $I$ has the finite intersection peoperty and so $A=\bigcap_{I^{\prime} \in F_{F}} I^{\prime} \neq \varnothing$. Thus $A$ is a compact ideal. If $a \in A$ then $a A \subset A$ is a compact ideal and so $a A=A$ which shows that $A$ is a group. If now $A_{1}$ is any other compact ideal and group of $S$ then $A_{1} A \subset A \cap A_{1} \neq \varnothing$ and if $a \in A \cap A_{1}$ then $A=$ $A a=A_{1} a=A_{1}$ which shows that $S$ contains exactly one ideal and compact group. Using Theorem (IV. 1) we get that $\operatorname{dim} J_{c} l(S)=1$ or that $C(S)$ admits a unique invariant mean. 


\section{REFERENCES}

1. A. D. Alexandroff, Additive set functions in abstract spaces (Russian), Math. Sbornic

8 (50) 1940, 307-348; 9 (51) 1941, 563-628; 13 (55) (1943), 169-238.

2. G. Birkoff, Compositio Math. (3) (1936), 424-430.

3. P. Civin-B. Yood, The second conjugate space of a Banach Algebra as an algebra, Pacific J. Math, 11 (1961), 847-870.

4. M. M. Day, Amenable semigroups, Illinois J. Math. 1 (1957), 509-544.

5. — Normed linear spaces, Berlin, Springer, 1958.

6. E. Granirer, On amenable semigroups with a finite dimensional set of invariant means I, II, Illinois J. Math. 7 (1963), 32-48 and 49-58.

7. - A theorem on amenable semigroups, Trans. Amer. Math. Soc. (to appear)

8. P. R. Halmos, Measure theory, New York, Van Nostrand (1959).

9. S. Kakutani, Über die Metrisation der topologixhen Gruppen Proc, Imp. Acad. Jap. 12 (1936), 82-84.

10. L. Kelley, General topology, New York, Van Nostrand, 1955.

11. Indar S. Luthar, Uniqueness of the invariant mean on an abelian semigroup, Illinois J. Math. 3 (1959), 28-44.

12. Uniqueness of the invariant mean on an abelian topological semigroup, Trans Amer. Math. Soc. (1962), 403-411.

13. Chivulkula R. Rao, In variant means on spaces of continuous or measuarable functions. thesis submitted to the University of Illinois, Urbana, Illinois.

14. W. G. Rosen, On invariant means over compact semigroups Proc. Amer. Math. Soc. 7 (1956), 1076-1082.

15. W. Rudin, Fourier Analysis on groups, Interscience Publishers, 1962.

16. R. J. Silverman, Invariant linear functions, Trans Amer. Math. Soc. 81 (1956), 411-424.

17. V. S. Varadarajan, Measures on topological spaces (Russian) Math. Sbornic, 55 (97) No. 1 (1961), 35-100.

18. A. Weil, Sur les espaces a structure uniforme at sur la topologic generale, Paris, Herman, Cie 1938.

19. K. Deleew, I. Glicksberg, Applications of almost periodic compactifications, Acta. Math. 105 (1961), 63-97.

20. E. Lyapin, Semigroups (Russian), Moskow, 1960.

21. R. Ellis, Locally compact transformation groups, Duke Math. J. 24 (1957), 119-120.

22. E. Hewitt, On two problems of Urisohn, Ann. of Math. (2) 47 (1946), 503-509.

23. W. W. Comfort and K. A. Ross, Pseudocompactness and uniform continuity in topological groups (to appear in the Pacific J. Math.)

THE UNIVERSITY OF ILLINOIS

URBANA, ILLINOIS 


\section{PACIFIC JOURNAL OF MATHEMATICS}

\section{EDITORS}

H. Samelson

Stanford University

Stanford, California

R. M. Blumenthal

University of Washington

Seattle, Washington 98105
J. Dugundu

University of Southern California Los Angeles, California 90007

*Richard Arens

University of California

Los Angeles, California 90024

\section{ASSOCIATE EDITORS}
E. F. BECKENBACH
B. H. NeumanN
F. WOLF
K. YOSIDA

\section{SUPPORTING INSTITUTIONS}

UNIVERSITY OF BRITISH COLUMBIA CALIFORNIA INSTITUTE OF TECHNOLOGY UNIVERSITY OF CALIFORNIA MONTANA STATE UNIVERSITY

UNIVERSITY OF NEVADA

NEW MEXICO STATE UNIVERSITY

OREGON STATE UNIVERSITY

UNIVERSITY OF OREGON

OSAKA UNIVERSITY

UNIVERSITY OF SOUTHERN CALIFORNIA
STANFORD UNIVERSITY

UNIVERSITY OF TOKYO

UNIVERSITY OF UTAH

WASHINGTON STATE UNIVERSITY

UNIVERSITY OF WASHINGTON

AMERICAN MATHEMATICAL SOCIETY CALIFORNIA RESEARCH CORPORATION SPACE TECHNOLOGY LABORATORIES NAVAL ORDNANCE TEST STATION 


\section{Pacific Journal of Mathematics}

\section{Vol. 15, No. $1 \quad$ September, 1965}

Donald Charles Benson, Unimodular solutions of infinite systems of linear

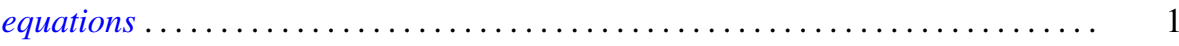

Richard Earl Block, Transitive groups of collineations on certain designs . . . . . . 13

Barry William Boehm, Existence of best rational Tchebycheff approximations .... . 19

Joseph Patrick Brannen, A note on Hausdorff's summation methods . . . . . . . . . . 29

Dennison Robert Brown, Topological semilattices on the two-cell ............ 35

Peter Southcott Bullen, Some inequalities for symmetric means . . . . . . . . . . 47

David Geoffrey Cantor, On arithmetic properties of coefficients of rational

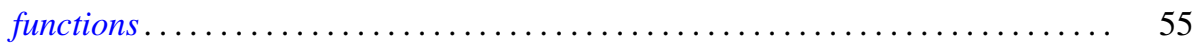

Luther Elic Claborn, Dedekind domains and rings of quotients . . . . . . . . . 59

Allan Clark, Homotopy commutativity and the Moore spectral sequence ........ 65

Allen Devinatz, The asymptotic nature of the solutions of certain linear systems of

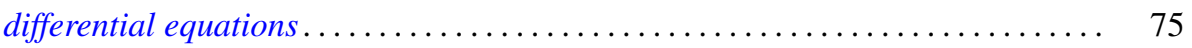

Robert E. Edwards, Approximation by convolutions ................... 85

Theodore William Gamelin, Decomposition theorems for Fredholm operators . . . . . 97

Edmond E. Granirer, On the invariant mean on topological semigroups and on

topological groups .................................. 107

Noel Justin Hicks, Closed vector fields . . . . . . . . . . . . . . . . . . . 141

Charles Ray Hobby and Ronald Pyke, Doubly stochastic operators obtained from

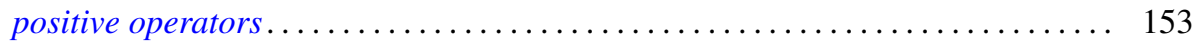

Robert Franklin Jolly, Concerning periodic subadditive functions . . . . . . . . . 159

Tosio Kato, Wave operators and unitary equivalence . . . . . . . . . . . . . . 171

Paul Katz and Ernst Gabor Straus, Infinite sums in algebraic structures . . . . . . . 181

Herbert Frederick Kreimer, Jr., On an extension of the Picard-Vessiot theory ...... 191

Radha Govinda Laha and Eugene Lukacs, On a linear form whose distribution is

identical with that of a monomial ......................... 207

Donald A. Ludwig, Singularities of superpositions of distributions . . . . . . . . . 215

Albert W. Marshall and Ingram Olkin, Norms and inequalities for condition

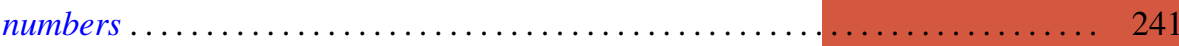

Horace Yomishi Mochizuki, Finitistic global dimension for rings . . . . . . . . . . 249

Robert Harvey Oehmke and Reuben Sandler, The collineation groups of division

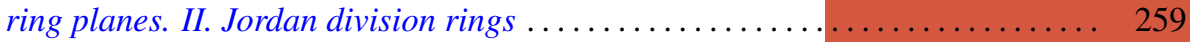

George H. Orland, On non-convex polyhedral surfaces in $E^{3} \ldots \ldots \ldots \ldots \ldots \ldots \ldots 267$

Theodore G. Ostrom, Collineation groups of semi-translation planes . . . . . . . . 273

Arthur Argyle Sagle, On anti-commutative algebras and general Lie triple

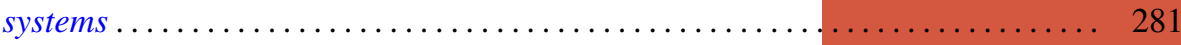

Laurent Siebenmann, A characterization of free projective planes . . . . . . . . . 293

Edward Silverman, Simple areas.................................. 299

James McLean Sloss, Chebyshev approximation to zero .................. 305

Robert S. Strichartz, Isometric isomorphisms of measure algebras . . . . . . . . . 315

Richard Joseph Turyn, Character sums and difference sets . . . . . . . . . . . . 319

L. E. Ward, Concerning Koch's theorem on the existence of arcs . . . . . . . . . . 347

Israel Zuckerman, A new measure of a partial differential field extension ......... 357 